\title{
Uncertainty about the Effort-Performance Relationship in Threshold-Based Payment Schemes
}

DOI:

10.1016/j.jhealeco.2018.09.003

Document Version

Accepted author manuscript

Link to publication record in Manchester Research Explorer

\section{Citation for published version (APA):}

Oxholm, A. S., Kristensen, S. R., \& Sutton, M. (2018). Uncertainty about the Effort-Performance Relationship in Threshold-Based Payment Schemes. Journal of Health Economics, 62, 69-83.

https://doi.org/10.1016/j.jhealeco.2018.09.003

\section{Published in:}

Journal of Health Economics

\section{Citing this paper}

Please note that where the full-text provided on Manchester Research Explorer is the Author Accepted Manuscript or Proof version this may differ from the final Published version. If citing, it is advised that you check and use the publisher's definitive version.

\section{General rights}

Copyright and moral rights for the publications made accessible in the Research Explorer are retained by the authors and/or other copyright owners and it is a condition of accessing publications that users recognise and abide by the legal requirements associated with these rights.

\section{Takedown policy}

If you believe that this document breaches copyright please refer to the University of Manchester's Takedown Procedures [http://man.ac.uk/04Y6Bo] or contact uml.scholarlycommunications@manchester.ac.uk providing relevant details, so we can investigate your claim.

\section{OPEN ACCESS}




\title{
Uncertainty about the Effort-Performance Relationship in Threshold-Based Payment Schemes
}

\author{
Anne Sophie Oxholm ${ }^{\mathrm{a}, *}$; Søren Rud Kristensen ${ }^{\mathrm{b}}$; Matt Sutton ${ }^{\mathrm{c}}$ \\ ${ }^{\text {a }}$ Danish Centre for Health Economics (DaCHE), Department of Public Health, University of Southern Denmark \\ Address: J.B. Winsløws Vej 9B, 5000 Odense C, Denmark \\ ${ }^{\mathrm{b}}$ Faculty of Medicine, Institute of Global Health Innovation, Imperial College London \\ Address: South Kensington Campus, London SW7 2AZ, United Kingdom \\ c School of Health Sciences, University of Manchester \\ Address: Oxford Road, Manchester M13 9PL, United Kingdom
}

\begin{abstract}
Incentive schemes often feature a threshold beyond which providers receive no additional payment for performance. We investigate whether providers' uncertainty about the relationship between effort and measured performance leads to financially unrewarded performance in such schemes. Using data from the British Quality and Outcomes Framework, we proxy general practitioners' uncertainty about the effort-performance relationship by their experience with the scheme and their span of control. We find evidence that providers respond to uncertainty by exerting financially unrewarded performance, suggesting that uncertainty may serve as a useful incentive tool to extract unrewarded performance for payers.
\end{abstract}

Key Words: Pay for performance; Uncertainty; Threshold; Provider behaviour

JEL Codes: I10

* Corresponding author. Email address: asoxholm@sdu.dk (AS Oxholm).

Acknowledgements: We are grateful to Per Andersen, Geir Godager, Hugh Gravelle, Lone Grønbæk, Lars Peter Raahave Østerdal, and Anthony Scott for helpful discussions. We would also like to thank participants, chairs and discussants at the Health Economists' Study Group Summer meeting 2014, the 35th Nordic Health Economists' Study Group meeting 2014, and at the 11th iHEA World Congress 2015 for helpful comments. Lastly, we wish to thank Yiu-Shing Lau for helping us with the statistical software. Any remaining errors are our own.

This research did not receive any specific grant from funding agencies in the public, commercial, or not-forprofit sectors 


\section{Introduction}

A key objective for payers in health care is to ensure that providers exert a sufficient amount of effort. However, providers' effort is usually unobservable to payers and thus impossible to contract on directly. Payers can instead use payment schemes that affect providers' willingness to exert effort (Chalkley and Malcomson, 1998; Ellis and McGuire, 1986; Holmstrom and Milgrom, 1991; Kaarboe and Siciliani, 2011). A popular approach is to pay providers for their performance on measurable process and outcome indicators (Cashin et al., 2014; Eijkenaar, 2013; Scott et al., 2018).

The design of performance-related payment schemes may feature upper thresholds beyond which providers are not paid for additional performance. Examples of such arrangements are target payments (Giuffrida et al., 1999) and piece-wise linear payment schedules with horizontal upper segments. Absolute upper thresholds are a common feature in international pay for performance schemes (for example the Quality and Outcomes Framework (QOF) in the United Kingdom (Gillam et al., 2012), the Primary Health Organization (PHO) Performance Programme in New Zealand (Buetow, 2008), the Primary Health Care Quality Bonus System (QBS) in Estonia (Cashin et al., 2014), a cancer screening program targeted primary care physicians in Ontario (Kiran et al., 2014), a pay for performance scheme targeted primary care in the Swedish county Skäne (Odesjo et al., 2015), and the Practice Incentive Programme (PIP) in Australia (Cashin et al., 2014)). Thresholds are popular because they provide a clear and simple standard of expected performance (Cromwell et al., 2011). These may not always induce maximum possible performance from providers, but may also save payers money if providers overachieve because they are uncertain about the relationship between their effort and measured performance. The scope of our study is to investigate whether financially unrewarded performance may be explained by uncertainty about the effort-performance relationship in threshold-based payment schemes.

We set up a theoretical model to explain why some providers exert financially unrewarded effort when exposed to a threshold-based payment scheme and the role of uncertainty about the effort-performance relationship. Our theoretical analysis follows the work by Zhou and Swan (2003) and Oxholm (2016), however we model a scheme resembling the Quality and Outcomes Framework. Similar to Oxholm (2016), we show that when providers are uncertain about the effort-performance relationship, those who perform above the upper threshold exert more effort compared to a situation where they can perfectly predict their own performance.

We test our theoretical predictions using data on Scottish general medical practices (GPs), which are remunerated based on the pay for performance scheme "the Quality and Outcomes Framework" (QOF) (Gillam et al., 2012; Allen et al., 2014). Neither GPs' effort level nor uncertainty can be measured directly in this scheme. We therefore use GPs' reported performance for QOF indicators as a proxy for their effort and exploit variation in their uncertainty arising from two sources: 1) experience-dependent uncertainty, which decreases as providers gain experience with each indicator; and 2) indicator-dependent uncertainty, which varies by scale of eligible patients and by the indicator type defined by the extent to which it is under the sole control of 
the practices or also depends on patient efforts. We estimate the importance of GPs' uncertainty at different parts of GPs' performance distribution using a quantile regression method allowing for fixed effects (Canay, 2011).

Our empirical results show that practice performance decreased above the upper threshold as practices gained experience in predicting their performance for the indicators over which they had more control (process indicators and large-scaled indicators) relatively to other indicators (outcome indicators and small-scaled indicators). In support of our hypothesis we thus find that uncertainty about the effort-performance relationship can explain unremunerated achievement. Providers' uncertainty may thus serve as an important incentive tool to extract unrewarded performance in threshold-based payment scheme.

In the following section we first introduce the Quality and Outcomes Framework, which is our case study. Section 3 presents our theoretical framework and hypotheses. Descriptions of the data we use are covered in Section 4. Section 5 and 6 covers our empirical strategy and results. Section 7 discusses this strategy and the results. The final section (8) contains our discussion of the results along with policy recommendations and suggestions for further research.

\section{Setting}

In 2004 a pay for performance scheme targeting GPs called the Quality and Outcomes Framework was introduced in the UK. The purpose of the QOF is to improve the quality of health care by measuring practice achievements against a set of indicators designed to promote good practice. The QOF initially consisted of 146 indicators measuring performance across four domains: clinical care, organisational standards, patient experience, and additional services. On the introduction of the QOF GPs saw their personal income increase by around $£ 25,000$, corresponding to a $30 \%$ increase on the previous year (Allen et al., 2014). Participation in the QOF is voluntary; however, 99.6\% of practices have signed up (Campbell et al., 2009).

Most of the clinical indicators in the QOF are based on the percentage of patients on whom a record is made, for whom a particular treatment is provided or an outcome standard is reached. As not all patients are treatable or willing to be treated, the QOF includes the possibility of exception reporting such that these patients do not count in the assessment of the practice's achievement. Practice $g$ 's achievement rate, $y_{i g t}$, for indicator $i$ at time $t$ is thus calculated as:

$$
y_{i g t}=\frac{N_{i g t}}{\text { prev }_{i g t}-e x c l_{i g t}-e x e p t_{i g t}}
$$

where $N_{i g t}$ is the number of patients fulfilling the indicator goal, previgt is the number of patients diagnosed with the condition (prevalence), excligt is the number of patients excluded from being a part of the indicator (e.g. based on their age), and except igt $_{\text {is }}$ the number of exception reported patients because they are untreatable or unwilling to be treated (Gravelle et al., 2010). 
Each QOF indicator is associated with a number of points. Whether a practice receives the maximum amount of points depends on the practice's performance, $y_{i g t}$, and the indicator's upper threshold level for performance. According to the DepartmentofHealth (2003) the upper threshold reflects the "maximum practically achievable level to deliver clinical effectiveness". The payer's intention behind setting an upper performance threshold is thus to reduce the risk that GPs inappropriately treat some patients (Doran et al., 2008, 2014; Roland, 2004). However, the thresholds are not set based on empirical evidence (Doran et al., 2014) and physicians are already allowed to exempt patients who are ineligible for treatment.

Payment per point is set to reflect the likely workload (Fleetcroft and Cookson, 2006). It depends on the proportion of patients diagnosed with the condition and the size of the registered population in the practice relative to the national average, which means that the payment per point may vary between practices and indicators. The calculation of practice $g$ 's performance payment, $Q O F_{i g t}$, for indicator $i$ at time $t$ is stated in Equations 2 - 4 (following Feng et al. $(2015))$ :

$$
\begin{gathered}
Q O F_{i g t}=0 \text { if } 0 \leq y_{i g t} \leq \text { lower }_{i t} \\
Q O F_{i g t}=r_{t} \cdot A D P F_{i g t} \cdot C P I_{g t} \cdot \pi_{i t}^{\text {max }} \frac{y_{i g t}-\text { lower }_{i t}}{\text { upper }_{i t}-\text { lower }_{i t}} \text { if }_{\text {lower }}<y_{i g t}<\text { upper }_{i t} \\
Q O F_{i g t}=r_{t} \cdot A D P F_{i g t} \cdot C P I_{g t} \cdot \pi_{i t}^{\text {max }} \text { if } \text { upper }_{i t} \leq y_{i g t} \leq 1
\end{gathered}
$$

As stated in equation 2 the practice does not receive any points if its performance, $y_{i g t}$, does not exceed the lower threshold, $l_{\text {ower }}$, for indicator $i$ at time $t$. The lower threshold for all indicators was $25 \%$ for the first two years and $40 \%$ thereafter. The upper threshold, upper ${ }_{i t}$, sets a cap on the maximum achievable points, $\pi_{i t}^{\max }$. The upper threshold was initially set between $55 \%$ and $90 \%$, such that achievement above this level is financially unrewarded, cf. equation 4 . Points for achievement between the lower and upper threshold are given proportionally to the achievement between the two thresholds, cf. equation 3 .

The practice's performance payment is based on a raw pounds-per-point value, $r_{t}$, which was $£ 75$ in $2004 / 5, £ 124.60$ in $2005 / 6$, and hereafter only slightly increased over time. To obtain practices' payment for an indicator the raw value per point is adjusted by two factors: the so-called Adjusted Disease Prevalence Factor, $A D P F_{i g t}$, and the Contractor Population Index, $C P I_{g t}$. ADPF measures the proportion of the practice's patients diagnosed with the relevant condition, where a high $A D P F$ value corresponds to a high disease prevalence among the practice's patients. $C P I$ is calculated as the size of the registered patient population relatively to the national average registered patient population (Guthrie et al., 2006), i.e. practices with large patient populations receive a higher pounds-per-point value.

In 2004, when the QOF was introduced, a large share of GPs performed above the upper achievement threshold for the indicators. The design of the payment scheme meant that these practices did not receive a financial reward for all their effort (Roland, 2004; Doran et al., 2008; 
Gravelle et al., 2010). Gravelle et al. (2010) estimated that Scottish practices in the second year of the scheme could have reduced the number of patients they treated by $11.8 \%$ without reducing their performance payment. The large amount of financially unrewarded performance in the QOF underlines the need to understand how payment caps affect providers' behaviour and more specifically the role uncertainty plays.

\section{A model of provider response to a threshold-based pay for performance scheme}

\subsection{The model}

Following the literature on physicians' decision-making (Dionne and Contandriopoulos, 1985), we assume that the practices maximise their utility by choosing the amount of effort to exert taking into account their own profit, their leisure time, and the health care given to their patients. Practice $g$ 's utility function is thus given by ${ }^{1}$ :

$$
u_{g}\left(e_{g}\right)=h_{g}\left(e_{g}\right)+m\left(e_{g}\right)-c_{g}\left(e_{g}\right)
$$

where $e_{g}>0$ measures the GP's supply of effort into the production of health care, $h_{g}$ is the GP's utility from providing health care, $m$ is the GP's revenue, and $c_{g}$ measures both the GP's direct and indirect costs.

Similar to other studies (Chalkley and Malcomson, 1998; Ellis and McGuire, 1986; Godager and Wiesen, 2013; Kaarboe and Siciliani, 2011; Makris and Siciliani, 2013), we acknowledge health care providers' altruistic and reputational gain from providing health care and allow this gain to differ between GPs by specifying the GP-specific utility term, $h_{g}$. Thus, the subscript "g" indicates that the utility gain from providing health care may vary across providers. We assume that the more effort GPs provide to patients, the more health care is provided and thus the higher is the GP's utility gain. As the first amount of effort most likely provides the most necessary treatment to patients, we further assume that the GP's marginal utility from providing health care is decreasing in effort, such that $h_{g}^{\prime}\left(e_{g}\right)>0$ and $h_{g}^{\prime \prime}\left(e_{g}\right)<0$.

We model GP's revenue, $m$, as a function of a payment scheme similar to the QOF, cf. Equations 2 - 4. For simplicity and because we are only interested in analysing performance above the upper threshold (financially unrewarded performance), we only include a single performance indicator and disregard the lower performance threshold in the QOF, which almost all GPs reach already the first year of the scheme (Gravelle et al., 2010). In addition we assume that the number of patients eligible for treatment, the disease prevalence rate, and the practice list size are fixed. Below the threshold the GP's payment is thus proportional to achievement, and above the threshold no additional financial reward is given for achievement, i.e. $m\left(e_{g}\right)=\beta e_{g}$ for

\footnotetext{
${ }^{1}$ For simplicity the arguments in the utility function are assumed additively separable.
} 
$0 \leq e_{g}<\tau$ and $m\left(e_{g}\right)=\beta \tau$ for $e_{g} \geq \tau$, where $\beta>0$ and $\tau$ is the minimum effort level required to reach the performance threshold.

The GP has both direct and indirect costs, $c_{g}$, from exerting effort to produce health care. The direct costs are human resources (wages) and equipment; these costs are assumed to increase proportionally with the amount of effort exerted. The GP also suffers a utility loss due to loss of leisure time. This indirect cost from providing treatment may differ across GPs. However, all GPs face a time constraint, which is captured by a strictly convex cost function, i.e. $c_{g}^{\prime}\left(e_{g}\right)>0$ and $c_{g}^{\prime \prime}\left(e_{g}\right)>0$.

\subsection{Base-case scenario: Financially unrewarded performance in a threshold- based pay for performance scheme}

We analyse GPs' financially unrewarded performance. We therefore begin by considering the benchmark case in which GPs perform strictly above the threshold, i.e. $e_{g}>\tau$. In this scenario GPs maximise their own utility by choosing effort such that ${ }^{2}$ :

$$
h_{g}^{\prime}\left(e_{g}\right)=c_{g}^{\prime}\left(e_{g}\right)
$$

According to Equation 6, a GP exerts effort above the performance threshold until the marginal reputational and altruistic gain from improving health care equals the marginal costs. Thus, the performance scheme may only affect GPs' willingness to exert effort above the threshold if it affects GPs' reputational or altruistic preferences.

\subsection{Uncertainty about the effort-performance relationship's effect on financially unrewarded performance}

As patients do not always comply with treatment and because it may be difficult for GPs to estimate their own achievement, GPs face uncertainty about the effort-performance relationship. This uncertainty may influence GPs' willingness to exert financially unrewarded performance. Using the above model we analyse the role this uncertainty plays in GP's decision-making when performing above the performance threshold. We build on work by Zhou and Swan (2003) and from Oxholm (2016) that model uncertainty in threshold-based contracts.

Let $\varepsilon$ be a stochastic shock to GP's performance occurring after effort is exerted. As the shock is unrelated to GP's behaviour, the shock does not affect the GP's cost or altruistic gain from providing health care - only the size of the monetary reward, $m\left(e_{g}+\varepsilon\right)$. The shock is assumed normally distributed with mean zero, $\varepsilon \sim N\left(0, \sigma^{2}\right)$, such that the GP expects to predict performance correctly. The normal distribution has previously been used to describe

\footnotetext{
${ }^{2}$ The functional form of $h_{g}\left(e_{g}\right)$ and $c_{g}\left(e_{g}\right)$ implies that $\lim _{e \rightarrow 0} h_{g}^{\prime}\left(e_{g}\right)-c_{g}^{\prime}\left(e_{g}\right) \rightarrow \infty$ and $\lim _{e \rightarrow \infty} h_{g}^{\prime}\left(e_{g}\right)-$ $c_{g}^{\prime}\left(e_{g}\right) \rightarrow-\infty$. We assume that $h_{g}(0)=0$ and $c_{g}(0)=0$ such that there is no health care and no disutility from exerting effort if no effort is exerted. We also assume that the physician always choose to exert a positive amount of effort $e_{g}>0$. This implies that the optimal effort level exists and is unique.
} 
agents' uncertainty about performance (Zhou and Swan, 2003; Eggleston, 2005). Let $\phi(\varepsilon)$ denote the probability density function of the shock $\varepsilon$. Then the probability that the GP does not reach the threshold is $P\left(e_{g}+\varepsilon<\tau\right)=\int_{-\infty}^{\tau-e_{g}} \phi(\varepsilon) d \varepsilon$. The GP's expected utility is defined as:

$$
E\left[u_{g}\left(e_{g}\right)\right]=h_{g}\left(e_{g}\right)+\int_{-\infty}^{\tau-e_{g}} \beta\left(e_{g}+\varepsilon\right) \phi(\varepsilon) d \varepsilon+\int_{\tau-e_{g}}^{\infty} \beta \tau \phi(\varepsilon) d \varepsilon-c_{g}\left(e_{g}\right)
$$

The GP chooses the effort level that maximises its expected utility. The first order condition for the GP's maximisation problem is thus given by:

$$
h_{g}^{\prime}\left(e_{g}\right)+\beta \int_{-\infty}^{\tau-e_{g}} \phi(\varepsilon) d \varepsilon=c_{g}^{\prime}\left(e_{g}\right)
$$

and states that the GP chooses its effort level such that the marginal utility gains from exerting effort equals the marginal utility costs. The marginal utility gains consists of two terms: the GP's altruistic and reputational gain from improving the health care and the marginal utility gain from effort associated with the incentive parameter $\beta$, which rewards an increase in performance below the threshold level.

Recall that we are interested in investigating financially unrewarded effort. We therefore focus on GPs exerting effort strictly above the threshold level. To analyse the effect of uncertainty about the effort-performance relationship we compare Equation 8 to the situation in which the GP is certain about own performance, i.e. Equation 6. The GP's marginal utility gain from exerting effort above the performance threshold is higher when there is uncertainty about own performance, while the marginal costs of effort are the same in the two scenarios. We therefore conclude that uncertainty about the effort-performance relationship leads GPs, who exert effort strictly above the threshold level, to increase effort compared to a situation of certainty. ${ }^{3}$

This result, however, does not imply that a GP who expects to reach the performance threshold will always decrease effort when uncertainty decreases marginally. In Appendix A we confirm Oxholm (2016)'s finding in our payment setting, namely that the size and sign of the effect of a marginal decrease in uncertainty depends on three factors: the GP's initial degree of uncertainty $^{4}$, the GP's initial effort level, and the threshold effort level. Thus, there exist extreme levels of uncertainty that yield a different response (see Appendix A).

\section{Data}

The data used for our empirical analysis were obtained from the Information Services Division of NHS National Services Scotland. Our sample consists of Scottish practices that report their

\footnotetext{
${ }^{3}$ The result is independent of the assumed distribution of the stochastic shock.

${ }^{4} \mathrm{GP}$ 's initial uncertainty level refers to his degree of uncertainty about the effort required to reach a given performance level.
} 
performance on the QOF. We chose Scottish practices because their policy environment was stable in the data period compared to their English counterparts for which other policy changes besides the QOF may have affected performance, for example practice-based commissioning, Alternative Providers of Medical Services, and re-organisation of Primary Care Trusts (Feng et al., 2015). An additional advantage of the Scottish data is a larger amount of variation in the size of practice populations (McLean et al., 2007), which we wish to exploit in our analysis.

We use practices' contract type and size of registered patient population recorded on an annual basis between 2004/05 and 2010/11. By indicator, we also observe information on the maximum achievable points, and lower and upper achievement threshold levels. Furthermore, we observe information on practices' reported achievement (measured as the percentage of eligible patients for whom the requirement is met), amount of earned points, number of eligible patients (measured as the denominator in the reported achievement), exclusion rate, and exception rate of patients for each indicator. These measures are all recorded on an annual basis.

To ensure that practices' experience with the scheme is the same across indicators, we include in the sample performance indicators that have existed since the introduction of the QOF in 2004/05. We further require that the indicators are evaluated based on threshold achievements and that the indicator description, maximum achievable points ${ }^{5}$, and upper threshold level remain constant until 2007/08 to ensure a sufficiently large data period for all indicators. Furthermore, register data on patient exception and exclusion reporting for the period must be publicly available. Based on these criteria, our data includes 23 indicators, of which two indicators decrease their maximal achievable points slightly the last two years of the data period (indicator BP04 and CHD06). ${ }^{6}$ Table 1 lists the indicators, we include in the analysis, together with their description and our classification of them as either process or outcome indicators, their mean performance the first year of analysis, and also their distribution of number of eligible patients across practices.

\footnotetext{
${ }^{5}$ We treat as trivial that the indicator BP05 increases its maximum achievable points by $1 / 56$ from 2005/06 to $2006 / 07$.

${ }^{6}$ In our analyses we run robustness check of our results where the data period is shortened to four years or where these two indicators are excluded.
} 


\begin{tabular}{|c|c|c|c|c|c|c|c|}
\hline \multirow[b]{2}{*}{ Indicator } & \multirow[b]{2}{*}{ Definition } & \multirow[b]{2}{*}{$\begin{array}{l}\text { Process } \\
\text { Indicator }\end{array}$} & \multirow[b]{2}{*}{$\begin{array}{l}\text { Upper } \\
\text { threshold }\end{array}$} & \multirow[b]{2}{*}{$\begin{array}{l}\text { Mean } \\
\text { performance }\end{array}$} & \multicolumn{3}{|c|}{ Number of eligible patients } \\
\hline & & & & & $\begin{array}{c}2 \overline{5 \text { th }} \\
\text { percentile }\end{array}$ & $\begin{array}{c}50 \text { th } \\
\text { percentile }\end{array}$ & $\begin{array}{c}75 \text { th } \\
\text { percentile }\end{array}$ \\
\hline ASTHMA06 & The percentage of patients with asthma who have had an asthma review in the previous 15 months & 1 & 70 & 82.36 & 156 & 262 & 378 \\
\hline BP04 & $\begin{array}{l}\text { The percentage of patients with hypertension in whom there is a record of the blood pressure in the previous } \\
9 \text { months }\end{array}$ & 1 & 90 & 93.58 & 369 & 647 & 981 \\
\hline BP05 & $\begin{array}{l}\text { The percentage of patients with hypertension in whom the last blood pressure (measured in the previous } 9 \\
\text { months) is } 150 / 90 \text { or less }\end{array}$ & 0 & 70 & 79.65 & 357 & 626 & 957 \\
\hline CHD02 & $\begin{array}{l}\text { The percentage of patients with newly diagnosed angina (diagnosed after } 1 \text { April 2003) who are referred for } \\
\text { exercise testing and/or specialist assessment }\end{array}$ & 1 & 90 & 91.96 & 18 & 33 & 54 \\
\hline CHD05 & $\begin{array}{l}\text { The percentage of patients with coronary heart disease whose notes have a record of blood pressure in the } \\
\text { previous } 15 \text { months }\end{array}$ & 1 & 90 & 97.74 & 124 & 218 & 328 \\
\hline CHD06 & $\begin{array}{l}\text { The percentage of patients with coronary heart disease in whom the last blood pressure reading (measured } \\
\text { in the previous } 15 \text { months) is } 150 / 90 \text { or less }\end{array}$ & 0 & 70 & 89.91 & 121 & 215 & 320 \\
\hline CHD07 & $\begin{array}{l}\text { The percentage of patients with coronary heart disease whose notes have a record of total cholesterol in the } \\
\text { previous } 15 \text { months }\end{array}$ & 1 & 90 & 94.54 & 123 & 214 & 322 \\
\hline CHD09 & $\begin{array}{l}\text { The percentage of patients with coronary heart disease with a record in the previous } 15 \text { months that aspirin, an } \\
\text { alternative anti-platelet therapy, or an anti-coagulant is being taken (unless a contraindication or side-effects } \\
\text { are recorded) }\end{array}$ & 1 & 90 & 95.46 & 124 & 215 & 327 \\
\hline COPD08 & $\begin{array}{l}\text { The percentage of patients with COPD who have had influenza immunisation in the preceding } 1 \text { September } \\
\text { to } 31 \text { March }\end{array}$ & 1 & 85 & 94.43 & 48 & 83 & 131 \\
\hline DM02 & The percentage of patients with diabetes whose notes record BMI in the previous 15 months & 1 & 90 & 96.20 & 117 & 204 & 305 \\
\hline DM05 & The percentage of patients with diabetes who have a record of HbA1c or equivalent in the previous 15 months & 1 & 90 & 97.94 & 118 & 207 & 305 \\
\hline DM09 & $\begin{array}{l}\text { The percentage of patients with diabetes with a record of the presence or absence of peripheral pulses in the } \\
\text { previous } 15 \text { months }\end{array}$ & 1 & 90 & 91.09 & 113 & 195 & 292 \\
\hline DM10 & The percentage of patients with diabetes with a record of neuropathy testing in the previous 15 months & 1 & 90 & 90.43 & 113 & 194 & 289 \\
\hline DM11 & The percentage of patients with diabetes who have a record of the blood pressure in the previous 15 months & 1 & 90 & 98.72 & 119 & 207 & 309 \\
\hline DM13 & $\begin{array}{l}\text { The percentage of patients with diabetes who have a record of micro-albuminuria testing in the previous } 15 \\
\text { months (exception reporting for patients with proteinuria) }\end{array}$ & 1 & 90 & 88.02 & 105 & 184 & 281 \\
\hline DM16 & The percentage of patients with diabetes who have a record of total cholesterol in the previous 15 months & 1 & 90 & 96.88 & 117 & 207 & 308 \\
\hline DM18 & $\begin{array}{l}\text { The percentage of patients with diabetes who have had influenza immunisation in the preceding } 1 \text { September } \\
\text { to } 31 \text { March }\end{array}$ & 1 & 85 & 93.08 & 103 & 179 & 268 \\
\hline STROKE05 & $\begin{array}{l}\text { The percentage of patients with TIA or stroke who have a record of blood pressure in the notes in the } \\
\text { preceding } 15 \text { months }\end{array}$ & 1 & 90 & 97.23 & 56 & 98 & 156 \\
\hline STROKE06 & $\begin{array}{l}\text { The percentage of patients with a history of TIA or stroke in whom the last blood pressure reading (measured } \\
\text { in the previous } 15 \text { months) is } 150 / 90 \text { or less }\end{array}$ & 0 & 70 & 88.67 & 55 & 97 & 152 \\
\hline STROKE07 & The percentage of patients with TIA or stroke who have a record of total cholesterol in the previous 15 months & 1 & 90 & 92.90 & 55 & 95 & 151 \\
\hline STROKE08 & $\begin{array}{l}\text { The percentage of patients with TIA or stroke whose last measured total cholesterol (measured in the previous } \\
15 \text { months) is } 5 \mathrm{mmol} / 1 \text { or less }\end{array}$ & 0 & 60 & 77.40 & 50 & 88 & 139 \\
\hline STROKE10 & $\begin{array}{l}\text { The percentage of patients with TIA or stroke who have had influenza immunisation in the preceding } 1 \\
\text { September to } 31 \text { March }\end{array}$ & 1 & 85 & 91.36 & 49 & 87 & 134 \\
\hline THYROI02 & $\begin{array}{l}\text { The percentage of patients with hypothyroidism with thyroid function tests recorded in the previous } 15 \\
\text { months }\end{array}$ & 1 & 90 & 97.10 & 89 & 167 & 262 \\
\hline
\end{tabular}

Notes: The table depicts indicators included in our analyses and describes whether they measure a process (value $=1$ ) or outcome (value $=0$ ) measure, their upper threshold for performance payment (in percentage), their mean performance in the first year of the analysis (in percentage), and the distribution of number of eligible patients in included practices (cut-off value for the 25th, 50th, and 75th percentile). We used a balanced panel with Scottish GPs with a GMS contract for the years 2005 to 2010. 
To avoid the influence of changes to the sample composition over time, we use a balanced panel of observations at the practice-indicator level between 2005/06 and 2010/11. ${ }^{7}$ The reason why we exclude the first year of the QOF is that the national average payment per point increased by $66 \%$ from $2004 / 05$ to $2005 / 06$, thereby increasing the incentive to exert effort substantially. We do not include data after 2010/11 because more than a third of the indicators (listed in table 1) no longer fulfil the requirements for inclusion in our analysis.

In Scotland approximately $88 \%$ of general practices work under the General Medical Services (GMS) contract (ISDScotland, 2013). GPs can alternatively sign up to a Personal Medical Services (PMS) contract or be owned and managed directly by the local Primary Care Organisation. We restrict our sample to practices that operate under a GMS contract because the other contract types may set quality and outcomes requirements conditional on local agreements and thus create different incentives than those induced for practices on GMS contracts.

For a given indicator we exclude practices with no eligible patients. For $0.6 \%$ of the observations the number of eligible patients is missing, because the population is less than five (in which case the actual number is confidential), we set the number of patients to two. In our data set the minimum (maximum) number of practices included for an indicator is 764 (780), corresponding to $85 \%(87 \%)$ of the Scottish practices with GMS contracts included in the QOF data set in $2005 / 06$.

In cases where the number of eligible patients is missing we observe the number of earned points but data on reported performance is missing. If the number of earned points is larger than zero and lower than the maximum achievable number of point, we can compute reported performance from information on the indicator's lower and upper threshold and the practice's earned points relative to the maximum number achievable. For observations with missing reported performance and zero points we impute reported achievement by the indicator-year average reported performance of practices below the lower threshold ${ }^{8}$. For observations with maximum points, we impute missing reported performance by the indicator-year average reported performance of practices above the upper threshold.

\section{$5 \quad$ Empirical approach}

We are interested in estimating the impact of GPs' uncertainty about the effort-performance relationship on reported performance among GPs who perform above an upper performance payment threshold. Our structural equation can be written as follows:

$$
y_{i g t}=\beta_{\sigma} \sigma_{i g t}^{2}+\beta_{x} X_{g t}+\alpha_{i g}+v_{t}+\varepsilon_{i g t}
$$

where $y_{i g t}$ is reported performance on indicator $i$ for practice $g$ in year $t$. The term $\sigma_{i g t}^{2}$

\footnotetext{
${ }^{7} \mathrm{As}$ it is only $11 \%$ of practices that drop out of the sample because of this requirement, we believe that our sample is representative of the population of practices in Scotland.

${ }^{8}$ In cases where there is no reported performance below the lower threshold, practices with missing data are excluded.
} 
is the GPs' degree of uncertainty about own effort-performance relationship, $X_{g t}$ is a vector of observable GP characteristics, and $\alpha_{i g}$ and $v_{t}$ are practice-indicator and year fixed effects. The coefficient of interest is $\beta_{\sigma}$, which measures the association between reported performance and uncertainty. As GPs' uncertainty is not directly observed, it is not straightforward to estimate this coefficient. In section 5.1 we present specific features of the QOF scheme, which allow us to identify different sources of GP uncertainty. The section discusses potential identification issues arising from these sources of uncertainty, and presents our estimation strategy to overcome these issues.

\subsection{Measuring uncertainty}

We do not observe a direct measure of the uncertainty faced by GPs. Instead, we rely on variation coming from different sources of uncertainty. Our first measure of uncertainty is based on the assumption that practices can exert more control over their level of performance when they gain experience with the scheme, i.e. GPs' uncertainty about their effort-performance relationship decreases over time. By exploiting the longitudinal aspect of the QOF data, we observe changes in GPs' reported performance as they learn about their own achievements.

We follow practices that are enrolled from the start of the scheme, which means that their experience is correlated with other potential time-related factors such as epidemics and health technological development. These other time-related factors may affect the practices' reported performance and potentially confound the effect of decreased uncertainty from experience. ${ }^{9}$ To avoid confounding we exploit variation from sources of uncertainty that varies across indicators, which allow us to identify the effect of uncertainty on performance.

The uncertainty that varies across indicators is designed to reflect the variance in performance that is not under the practices' control. Following Baker (2002), we assume that the extent of uncertainty is positively correlated with the extent to which other agents' actions influence the performance measure. This uncertainty is related to the characteristics of the QOF indicators.

Our first measure of uncertainty that varies across indicators is the type of indicator, i.e. whether the indicator is an outcome or process measure. We posit that intermediate health outcome measures (outputs), such as achievement of blood pressure control limits, are more influenced by patients than the process measures (inputs), e.g., whether the practice records the patients' blood pressure.

Our second measure of uncertainty that varies across indicators is the scale of eligible patients. We assume that for a given practice, the degree of uncertainty is lower when mean performance is measured over more patients ("trials"). This argument is based on the law of large numbers, i.e., as more trials are performed, the average of the results should be closer to the expected value. As GPs may over-diagnose patients (Gravelle et al. 2010), the scale of eligible

\footnotetext{
${ }^{9}$ We do not believe it is feasible to exploit variation from practices entering into the scheme at a later time, because these practices most likely have different characteristics than other practices, and there are too few of these practices.
} 
patients could potentially be endogenously determined, i.e. the scale may be correlated with the predictability of the indicator. To alleviate this issue, we identify three "pairs" of process and outcome indicators with similar definitions but different target groups and thus different scales of eligible patients. Indicators STROKE05 ("small-scaled, process") and STROKE06 ("smallscaled, outcome") were targeted at the smallest population, TIA and stroke patients. Indicators CHD05 ("medium-scaled, process") and CHD06 ("medium-scaled, outcome") were targeted at a medium-size population, patients with coronary heart disease. Indicators BP04 ("Large- scaled, process") and BP05 ("Large-scaled, outcome") were targeted at the largest population, patients with hypertension (see table 1).

Our identification of the effect of uncertainty hinges on the assumption that different time trends in performance between more certain and less certain indicators are caused by reductions in uncertainty as GPs' gain experience with the scheme. Thus, we assume that differences in the effect of for example GPs' altruistic concerns across indicators are time-invariant. Section 7.2 provides a discussion of this assumption.

\subsection{Estimating the effect of uncertainty on reported performance}

The analysis of movements over time in a practice's reported performance requires careful consideration, because the movements may reflect other factors than uncertainty. One factor could be the bounded nature of the performance measure. As the performance score falls in the range 0-100\%, practices' potential for improvement over time may differ across indicators. Table 1 shows that the upper threshold level for performance payment is on average higher for process indicators than for outcome indicators. This difference is also reflected by a higher average performance score on process indicators. Thus, practices' potential for improvement over time is more limited on process indicators relatively to outcome indicators.

To address the potential issue associated with the bounded nature of our dependent variables, we follow the method proposed by Stevens et al. (2016) who suggest using the empirical logit transformation to linearise performance indicators measured on a percentage scale. The standard logit transformation of the performance $p$ is defined as $\operatorname{logit}(p)=\ln (p /(1-p))$ and expands any scale bounded between 0 and 1 to a range of minus infinity to plus infinity. As minus and plus infinity is interpreted by statistical packages as missing values, Stevens et al. (2016) suggests the use of the empirical logit transformation at the floor (0) and ceiling (1). This transformation is defined as $\operatorname{logit}(p)=\ln [(p+0.5 / n) /(1-p+0.5 / n)]$, where $n$ is the denominator of the performance ratio. Henceforth, we therefore set $y$ equal to $\operatorname{logit}(p)$. The transformation thus addresses the potential non-linearity in performance indicators due to differences in levels of effort needed for improving performance near the performance ceiling relative to the middle of the distribution.

The analysis of movements over time in a practice's reported performance may also reflect regression to the mean, i.e., that unusually high or low performance tends to be followed by performance that is closer to the mean (Barnett et al., 2005), rather than changes in performance caused by a decrease in uncertainty over time. One should therefore be careful with running a 
standard OLS panel regression. To alleviate the potential issue of regression to the mean, we estimate the development in GPs' reported performance over time at different points in the conditional performance distribution using quantile regression which, as opposed to running separate regressions at different parts of the distribution, uses all available data to estimate coefficients at different quantiles. In addition, quantile regression does not make assumptions about regression errors, and is more robust to outliers than mean regression (Cameron and Trivedi, 2008).

To control for time invariant practice-indicator level heterogeneity, we estimate quantile regression models with practice-indicator fixed effects, following the method proposed by Canay (2011). This method has previously been applied in a health-care setting, for example by Moscelli et al. (2018) to estimate differences in the socio-economic gradient at different points of the waiting time distribution. Canay's method consists of two-steps: In the first step we estimate practice-indicator fixed effects at the conditional mean of the reported performance. In the second step we first calculate adjusted performance scores by subtracting the predicted fixed effects from the dependent variable (reported performance) and then run quantile regression of the adjusted performance scores on the same set of regressors as in the first step. This two-step approach relies on the assumption that the practice-indicator fixed effects are independent of the location on the performance distribution, i.e. the fixed effects reflect location shifts that are independent of the quantile (Canay, 2011).

We specify two different models for reported performance at quantile $(q)$ as a function of the degree of uncertainty about the effort-performance relationship. We use the first model to estimate differences in reported performance over time by indicator type (process versus outcome). Equation 10 specifies for indicator $i$ and practice $g$ with experience $t$ the $q t h$ conditional quantile of the transformed performance scores $\left(\tilde{y}_{i g t}=y_{i g t}-\alpha_{i g}\right)^{10}$ :

$$
\begin{array}{r}
\text { Quant }^{q}\left(\tilde{y}_{i g t} \mid X_{i g t}\right)=\beta_{0}^{q}+\beta_{1}^{q} \text { experience }_{t}+\beta_{2}^{q} \text { experience }_{t} \cdot \text { process }_{i} \\
+\beta_{4}^{q} \text { listsize }_{g t}+\beta_{5 t}^{q} \text { prevrate }_{i g t}+\beta_{6}^{q} \text { exclrate }_{i g t}+\beta_{7}^{q} \text { exceptrate }_{i g t}+\varepsilon_{i g t}^{q}
\end{array}
$$

where we assume $\operatorname{Quant}^{q}\left(\varepsilon_{i g t}^{q} \mid X_{i g t}\right)=0 . \beta_{0}^{q}$ is a constant, experience e $_{\text {a }}$ linear year term, process $_{i}$ is a dummy variable taking values one for a process indicator and zero for an outcome indicator, listsize $_{g t}$ measures practice list size, prevrate $_{i g t}$ reports the disease prevalence rate ${ }^{11}$, exclrate $_{\text {igt }}$ measures the share of patients excluded from the performance measure, exceptrate $e_{\text {igt }}$ measures the share of patients exempted from the performance measure.

The estimate $\hat{\beta}_{2}^{q}$ is of particular interest. It measures how GPs' experience with process indicators influences reported performance compared to the influence from experience with outcome indicators. Our hypothesis is the following: As GPs performing above the upper threshold gain

\footnotetext{
${ }^{10} \alpha_{i g}$ is fixed effects on practice-indicator level obtained by regressing (OLS) $X_{i g t}$ on $y_{i g t}$.

${ }^{11}$ We allow for the effect of prevalence rate to change over time such that we capture changes in the relationship between prevalence and payment in 2009/10.
} 
experience, they reduce their achievement on process indicators (easier to control) relatively to outcome indicators, i.e. $\hat{\beta}_{2}^{q}<0$. Thus, this estimate reveals the importance of uncertainty about the relationship between own effort and performance for practices on the qth quantile of GPs' performance distribution.

We run three versions of model 1 using the transformed performance scores. The first version (model 1a) compares process and outcome indicators with the same upper threshold level for performance payment. As it is only at the threshold level of $70 \%$ outcome and process indicators share a common threshold, this model relies on four indicators. Thus, for this version of the model, we do not expect differences in the potential for improvement across indicator type to drive our results. ${ }^{12}$ The second and third version of the model (model $1 \mathrm{~b}$ and $1 \mathrm{c}$ ) include process indicators and outcome indicators that differ in upper threshold levels. We control for these differences by including the explanatory variable experience $e_{t} \cdot$ upper $_{i}$, where upper $_{i}$ is a set of

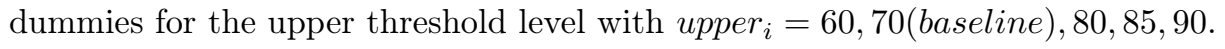

The difference between model $1 \mathrm{~b}$ and $1 \mathrm{c}$ is the included process indicators. As improvement in process indicators may spillover to outcome indicators, model $1 \mathrm{~b}$ includes only process indicators that are a part of a disease domain with no outcome indicators, whereas model 1c includes all process indicators listed in table 1. Thus, in the presence of spillover effects, we expect that practices have less control over process indicators included in model 1c than in model 1b, because overachievement on some of these indicators is required to achieve the (more uncertain) outcomes, i.e. $\hat{\beta}_{2, \text { model } 1 b}^{q}<\hat{\beta}_{2, \text { model } 1 \text { c }}^{q}$

We also consider the scale of eligible patients as a source of uncertainty. We use the second model (Equation 11) to estimate differences in reported performance over time by practices' scale of eligible patients. Equation 11 specifies the $q$ th conditional quantile of the transformed performance score on indicator $i$ for practice $g$ at experience $t$ :

$$
\begin{aligned}
\text { Quant }^{q}\left(\tilde{y}_{i g t} \mid X_{i g t}\right)= & \gamma_{0}^{q}+\gamma_{1}^{q} \text { experience }_{t}+\gamma_{2}^{q} \text { experience }_{t} \cdot \text { large }_{i}+\gamma_{3}^{q} \text { experience }_{t} \cdot \text { medium }_{i} \\
& +\gamma_{5}^{q} \text { listsize }_{g t}+\gamma_{6 t}^{q} \text { prevrate }_{i g t}+\gamma_{7}^{q} \text { exclrate }_{i g t}+\gamma_{8}^{q} \text { exceptrate }_{i g t}+\varepsilon_{i g t}^{q}
\end{aligned}
$$

where we assume $\operatorname{Quant}^{q}\left(\varepsilon_{i g t}^{q} \mid X_{i g t}\right)=0$. large $_{i}$ and medium $_{i}$ are binary indicators, and thus the $\operatorname{small}_{i}$ (most uncertain) indicator, is our baseline.

The estimates of particular interest are $\hat{\gamma}_{2}^{q}$ and $\hat{\gamma}_{3}^{q}$, which measure how GPs' experience with a large-scaled indicator and medium-scaled indicator affect reported performance compared to the effect of experience with a small-scaled indicator. We hypothesise that practices above the upper threshold reduce their reported performance for less uncertain indictors (large-scaled indicators

\footnotetext{
${ }^{12}$ In fact, in this case the average initial performance for the process indicator is slightly lower than for the average outcome indicators with an upper threshold level of $70 \%$, which implies that there is initially a slightly larger potential for improvement for the more certain (process) indicator. Thus, a relative decline in performance with experience on the process measure compared to the outcome measures is not caused by the limited performance range.
} 
and medium-scaled indicators) compared to more uncertain indicators (small-scaled indicators) as they gain experience. That is the estimate $\hat{\gamma}_{2}^{q}$ for a large-scaled indicator is expected to be smaller than for a medium-scaled indicator $\hat{\gamma}_{3}^{q}$ and that these these two estimates are expected to be negative because the reference category is a small-scaled indicator, i.e. $\hat{\gamma}_{2}^{q}<\hat{\gamma}_{3}^{q}<0$. As certainty of indicators also differ in regards to their indicator type, we run two versions of model 2. Model $2 \mathrm{a}$ includes only process indicators and model $2 \mathrm{~b}$ includes only outcome indicators.

Following other studies applying quantile regressions (see for example Chen et al. (2014)), we estimate the two models at the $10 t h, 25 t h, 50 t h, 75 t h$, and 90thquantile. This enables us to study the effects of uncertainty at both the bottom and top of the performance distribution. We use a bootstrap procedure to estimate standard errors (400 replications), computing both steps in Canay's two-step estimation procedure for each bootstrapped sample, which is sampled in clusters (block bootstrapped) at the practice-indicator level. We estimated all models with 400 bootstrap replications because doubling the number of bootstraps from 200 to 400 had negligible effects on the standard errors.

\section{$6 \quad$ Results}

\subsection{Descriptive analysis}

Before turning to the regression results, we present a descriptive analysis of the amount of financially unrewarded achievement. By indicator type and experience with the scheme, Table 2 presents the distribution of practice performance given by the distance to the upper performance threshold. ${ }^{13}$ From this we make two observations: First, the share of practices that overachieve by 5 percentage points or more is larger for outcome indicators than for process indicators. Second, achievement above the upper threshold tends to decrease with experience on the process indicators, moving practices from the $5 \%+$ category, to the $[0,5 \%]$ overachievement category. The same is not true for outcome indicators, on which the percentage of overachievement in the top category has increased slightly over time. Both observations are consistent with our expectations that for process indicators, which are easier for GPs to control, the amount of financially unrewarded achievement will decrease with experience at a faster rate compared to outcome indicators. Yet based on these simple descriptive statistics we cannot rule out other explanations, such as more GPs reaching the upper threshold for process indicators as experience increases, changes in practice list size, the disease prevalence rate and so on.

The scale of eligible patients is another source of uncertainty we consider in this paper. Next, we therefore examine reported performance over time by practices' scale of eligible patients for different indicator types. We consider three pairs of process and outcome variables, which differ only in their target groups. The target group for patients suffering from hypertension

\footnotetext{
${ }^{13}$ Most of the process indicators have 10 percentage points of financially unrewarded performance. To ensure that we can analyse behaviour close to and far from the threshold we analyse behaviour 0-5 percentage points from the threshold as "close to the threshold" and 5 or more percentage points from the threshold as "far from the threshold".
} 
Table 2: Percentages of observations By Distance to UpPer PERFormance threshold BY EXPERIENCE AND TYPE OF INDICATOR

\begin{tabular}{|c|c|c|c|c|c|c|c|c|c|c|c|c|c|}
\hline \multirow{2}{*}{\multicolumn{2}{|c|}{$\begin{array}{l}\text { Performance distance } \\
\text { from upper threshold }\end{array}$}} & \multicolumn{6}{|c|}{$\frac{\text { Outcome indicators }}{\text { Experience (years) }}$} & \multicolumn{6}{|c|}{$\frac{\text { Process indicators }}{\text { Experience (years) }}$} \\
\hline & & 1 & 2 & 3 & 4 & 5 & 6 & 1 & 2 & 3 & 4 & 5 & 6 \\
\hline$[-100 \%,-5 \%]$ & $\%$ of obs. & 2 & 1 & 0 & 0 & 0 & 0 & 6 & 4 & 3 & 2 & 2 & 2 \\
\hline$(-5 \%, 0 \%]$ & $\%$ of obs. & 2 & 1 & 0 & 0 & 1 & 1 & 6 & 4 & 3 & 2 & 3 & 3 \\
\hline$(0 \%, 5 \%]$ & $\begin{array}{l}\% \text { of obs. } \\
\% \text { of FUP }\end{array}$ & $\begin{array}{l}6 \\
7\end{array}$ & $\begin{array}{l}3 \\
3\end{array}$ & $\begin{array}{l}3 \\
3\end{array}$ & $\begin{array}{l}2 \\
2\end{array}$ & $\begin{array}{l}2 \\
2\end{array}$ & $\begin{array}{l}2 \\
2\end{array}$ & $\begin{array}{l}22 \\
26\end{array}$ & $\begin{array}{l}23 \\
25\end{array}$ & $\begin{array}{l}25 \\
27\end{array}$ & $\begin{array}{l}26 \\
27\end{array}$ & $\begin{array}{l}27 \\
28\end{array}$ & $\begin{array}{l}29 \\
31\end{array}$ \\
\hline$(5 \%, 100 \%]$ & $\begin{array}{l}\% \text { of obs. } \\
\% \text { of FUP }\end{array}$ & $\begin{array}{l}89 \\
93\end{array}$ & $\begin{array}{l}95 \\
97\end{array}$ & $\begin{array}{l}97 \\
97\end{array}$ & $\begin{array}{l}97 \\
98\end{array}$ & $\begin{array}{l}97 \\
98\end{array}$ & $\begin{array}{l}97 \\
98\end{array}$ & $\begin{array}{l}66 \\
74\end{array}$ & $\begin{array}{l}69 \\
75\end{array}$ & $\begin{array}{l}69 \\
73\end{array}$ & $\begin{array}{l}69 \\
73\end{array}$ & $\begin{array}{l}69 \\
72\end{array}$ & $\begin{array}{l}66 \\
69\end{array}$ \\
\hline
\end{tabular}

Notes: The table summarises practices' achievements' distance from the upper threshold for included indicators (in percentage points) by experience. The indicators are divided into outcome and process indicators, as shown in table 1. We define financially unrewarded performance (FUP) as reported achievement, exceeding the upper threshold. We use a balanced data set with Scottish GPs with a GMS contract for the years 2005 to 2010. Each year includes 3,109 observations for outcome indicators and 14,708 observations for process indicators.

(BP-indicators) is on average (across all years) around three times larger than for coronary heart disease patients (CHD-indicators) and almost seven times larger than for stroke patients (STROKE-indicators), cf. table 1. Figure 1 presents the distribution of reported performance on these indicators by experience level. Performance on the indicator large-scaled, process (BP04) is expected to be less uncertain than performance on the other indicators, because it is both larger scale and of the process type. ${ }^{14}$ As seen from Figure 1, the reported performance distribution of the indicator "large-scaled, process" seems to be narrowing with experience compared to the other indicators, suggesting that uncertainty about performance on this indicator decreases relative to the uncertainty about performance on the other indicators. Thus, the patterns we observe in our data are consistent with the expectation that as physicians gain experience, this indicator is easier to control compared to the other indicators.

\footnotetext{
${ }^{14}$ The drop in the performance distribution for the indicators "small-scaled, process" and "medium-scaled, process" depicted in figure 1 may be explained by the fact that $53 \%$ and $18 \%$ of practices have fewer than 100 eligible patients. For these practices it is mathematically impossible to obtain a performance score between $99 \%$ and $100 \%$.
} 
Figure 1: Kernel distribution of distance to upper performance threshold by experience and indicator type
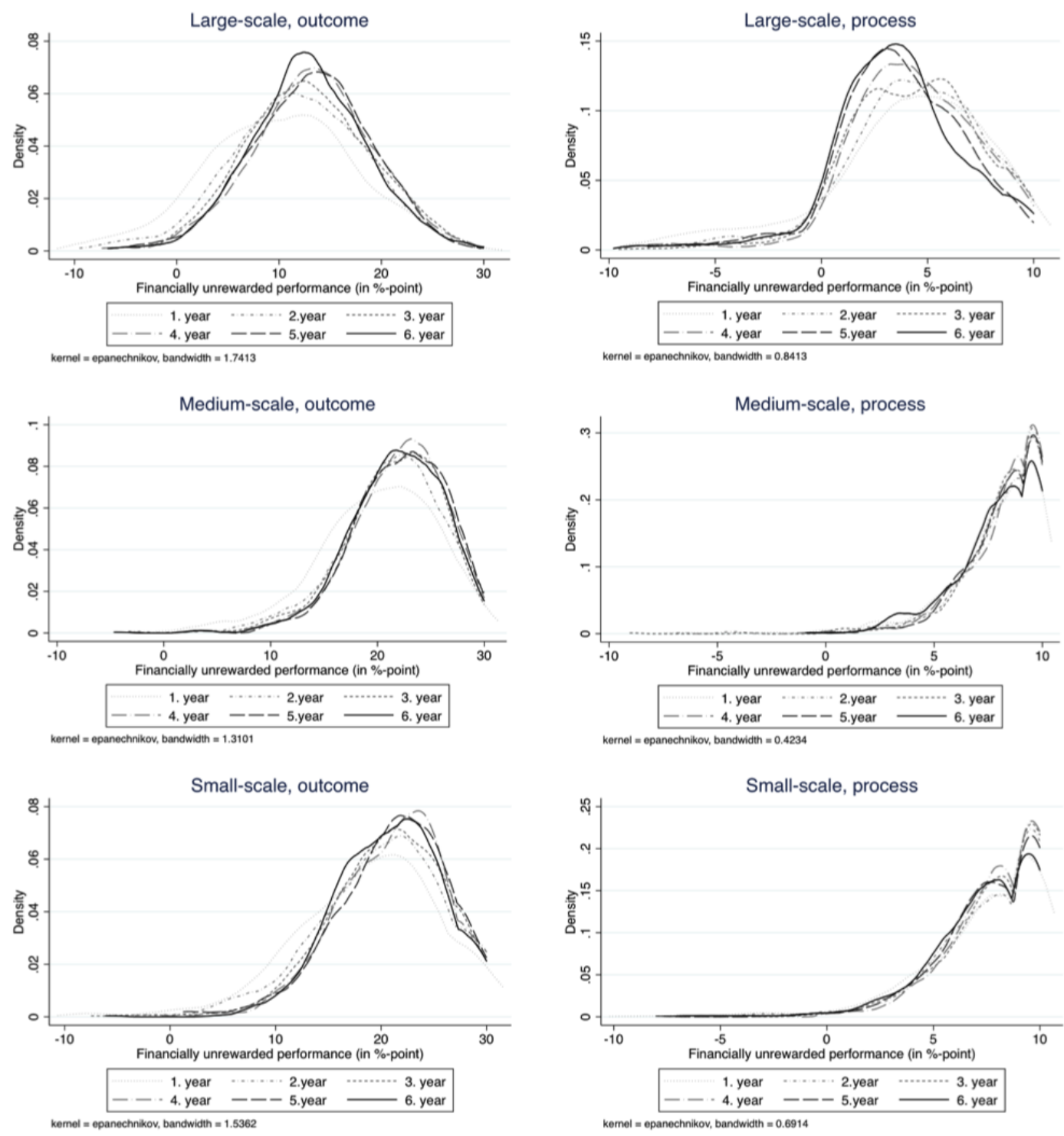

Notes: The figures depict the Epanechnikov kernel distribution of distance to upper performance threshold (in percentage points) by experience for each of the following indicators: "large-scaled, outcome" (BP05) and "large-scaled, process" (BP04). "medium-scaled, outcome" (CHD06) and "medium-scaled, process" (CHD05). "smallscaled, outcome" (STROKE06) and "small-scaled, process" (STROKE05). Only data for Scottish GPs with a GMS contract for the years 2005 to 2010 are included. 
Our observations in table 2 and figure 1 may, however, also be explained by other factors than physicians' uncertainty about the effort-performance relationship such as changes in the disease prevalence rate, patient list size, and the upper threshold level for performance payment. We therefore turn to regression analysis to estimate the importance of uncertainty on financially unrewarded performance.

\subsection{Regression results}

We now turn to the results from our regression analyses. Table 3 presents the regression estimates from model 1 (equation 10) for the effects of experience and indicator type on reported performance. The estimates are back-transformed using the method proposed by Stevens et al. $(2016) \cdot{ }^{15}$

The results show that practices at each of the five quantiles of the performance distribution (except the 90th quantile for model 1c) increase their performance on the outcome indicators as they gain experience with the scheme. As we cannot control for time specific effects (technological improvements, population health etc.) this change over time may be a result of other factors than uncertainty. To isolate the effect of uncertainty on performance, we consider the difference in achievement as experience increases for process indicators that are easier to control (less uncertain) than outcome indicators. The results show that practices decrease their performance on the process indicators over time relatively to outcome indicators at all parts of the performance distribution (except the $90 t h$ percentile for model $1 \mathrm{c}^{16}$ ). As $95 \%$ of practices perform above the upper threshold on process indicators already the first year of included experience, our findings (except for the top performing practices) support our hypothesis that GPs reduce their overachievement for indicators that are easier to control. For the lower part of the performance distribution the relative decrease in reported performance over the six-year experience period is more than one percentage point per year. As expected, the relative decrease in performance is less pronounced in model 1c than in the two other models, which include only process indicators that are a part of a disease domain with no outcome indicators, i.e. the link to a more uncertain outcome is weaker.

To account for the greater potential for continued improvement on indicators with lower thresholds in model $1 \mathrm{~b}$ and model $1 \mathrm{c}$, these models include an interaction term for the threshold level and GP's experience. ${ }^{17}$ As it is only for an upper threshold of $70 \%$ that outcome and process indicators share the upper threshold, identification of the process-experience interaction relies on comparison of one process indicator with three outcome indicators. As a robustness

\footnotetext{
${ }^{15}$ Stevens et al. (2016) propose that the change in the performance $p$ per unit increase in $x$ may be calculated as $\left.[\exp (-\beta)(1-p) / p+1)^{-1}-p\right]$, where $\beta$ is the estimate from the regression using the transformed performance score and a anchor value is assumed for $p$. We assume that the anchor value for $p$ is the associated quantile performance stated in Appendix B.

${ }^{16}$ As the raw performance for the 90 th percentile is $100 \%$, the back-transformed estimates are very close to zero.

${ }^{17}$ As there may be an issue with collinarity between the interaction terms experience $\cdot$ process and experience . upper in model $1 \mathrm{~b}$ and 1c, we run robustness checks in which we control for the upper threshold using a linear function of the threshold level interacted with experience. Our findings confirm our previous results, cf. table 5.
} 
Table 3: THE EFFECTS OF EXPERIENCE AND INDICATOR TYPE ON PRACTICE REPORTED PERFORMANCE

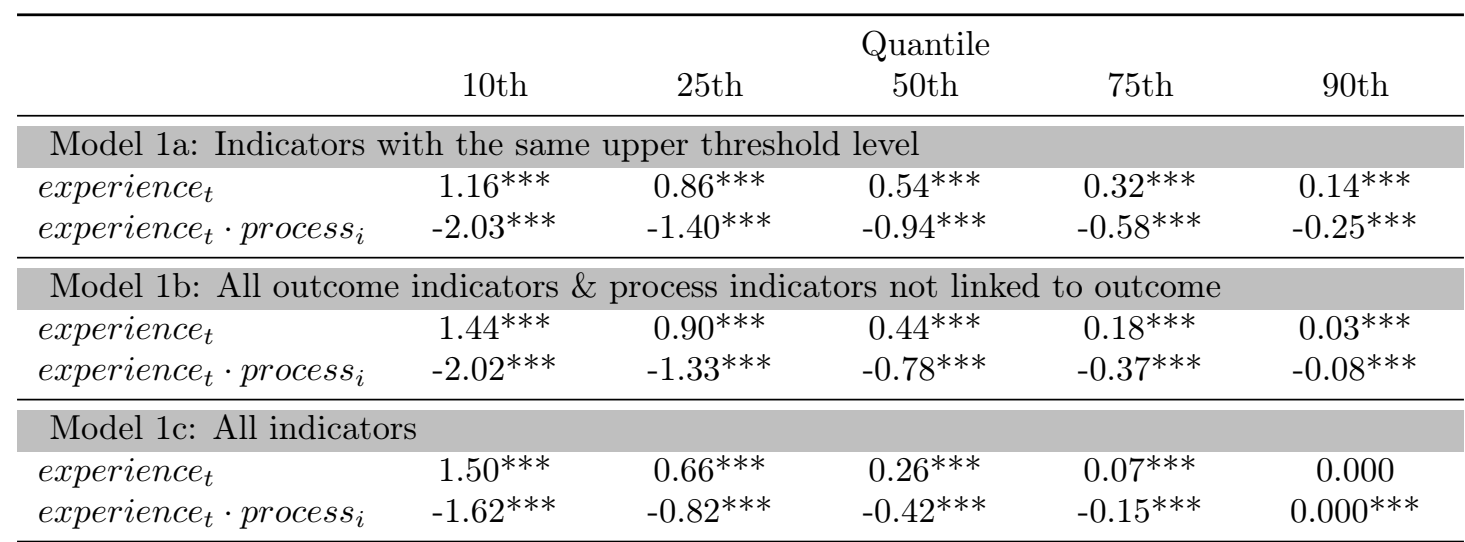

Notes: The columns contain estimated coefficients (in percentage points) from five quantile regressions run for three different versions of model 1 (1a, 1b, and 1c). All versions regress practice reported performance in the Quality and Outcomes Framework on experience, experience interacted with indicator type (a dummy equalling one for process indicator), list size, prevalence, prevalence rate interacted with year dummies, exclusion rate, and exception rate. In addition, model $1 \mathrm{~b}$ and model $1 \mathrm{c}$ control for experience interacted with dummies for the size of the upper threshold level (baseline 70\%). The performance scores are transformed using a logit transformation and adjusted for practice-indicator fixed effects obtained by OLS regression. This table presents the back-transformed estimates (see Appendix B for the nonback-transformed estimates). The three versions of model 1 include the indicators listed in table 1. However, model 1a only includes indicators with an upper threshold level of $70 \%$, model $1 \mathrm{~b}$ includes all outcome indicators and process indicators not linked to outcome indicators within a disease domain (ASTHMA06, COPD08, THYROI02), and model 1c includes all indicators. Only data for Scottish GPs with a GMS contract for the years 2005 to 2010 are included. The numbers of observations are 18,624, 32,574, and 106,902 for model 1a, 1b, and 1c, respectively. Standard errors are obtained by bootstrapping (400 rep) and reported in Appendix B. ${ }^{*} \mathrm{p}<0.10,{ }^{* *} \mathrm{p}<0.05,{ }^{* * *} \mathrm{p}<0.01$. 
check we estimate the two versions of model 1 without controlling for the upper threshold over time, and reassuringly, reach similar results, cf. section 6.3 .

In model 2 (Equation 11) we test for differences in the effect of scale of eligible patients on reported performance for process and outcome indicators as the physicians gain experience with the scheme. For this purpose, we use data for three pairs of similar process and outcome indicators; across pairs the indicators differ only by the scale of eligible patients (target groups). One of the advantages of comparing similar indicators is that we need not take into account potential issues caused by differences in indicator-specific characteristics. We estimate two versions of the model; model $2 \mathrm{a}$ includes only process indicators and model $2 \mathrm{~b}$ includes only outcome indicators. Table 4 presents the regression results for different locations in the performance distribution (quantiles $10 t h, 25 t h, 50 t h, 75 t h$ and $90 t h)$.

At each of the quantiles of the performance distribution in model $2 \mathrm{~b}$ and the lowest quantiles (10th and 25th) in model 2a, practices increase reported performance for the baseline indicator "small-scaled" as they gain experience with the scheme. We expect that an increase in the scale of eligible patients reduce physicians' uncertainty about their effort-performance relationship, making practices reduce financially unrewarded performance more with experience. We find that the effect of experience on the large-scaled indicator compared to the small-scaled is negative across the bottom four quantiles (insignificant for the 10th and 25th) for process indicators (model 2a) and for the top three quantiles (insignificant for the 50th) for outcome indicators (model 2b). ${ }^{18}$ For the medium-scaled indicators the relative decrease compared to the smallscaled indicator is less pronounced and only statistically significant for a few quantiles. Thus, the uncertainty arising from scale appears to have the strongest effect when we compare indicators with a large difference in size of target groups.

Overall our findings for both model 1 and 2 supports our hypothesis that overachievement decreases when uncertainty about the effort-performance decreases.

\subsection{Robustness of the results}

We perform several additional analyses to assess the robustness of our results. First, to check whether our results for model $1 \mathrm{~b}$ and $1 \mathrm{c}$ suffer from collinarity between the interaction terms experience · process and experience - upper, we estimate both versions of model 1 , letting the upper threshold level enter linearly in the interaction with experience. Second, we estimate both versions of the model without controlling for the upper threshold over time. Table 5 (panel I and II) presents estimates from these two analyses. Reassuringly, the results are qualitatively similar to our main results.

Next, we investigate whether the results for model 1c change when we exclude the indicators BP04 and CHD06, for which the maximum achievable points decrease by two points in the last

\footnotetext{
${ }^{18}$ For the large-scaled, outcome indicator for the lowest quantile (10th) the performance increases relatively to the small-scaled, outcome indicator. This increase may be attributed to a relatively larger share of practices initially performing below the upper threshold on the large-scaled indicator, cf. figure 1.
} 
Table 4: The effects of Experience, Patient scale And indicAtor type on practice REPORTED PERFORMANCE

\begin{tabular}{|c|c|c|c|c|c|}
\hline & \multicolumn{5}{|c|}{ Quantile } \\
\hline & 10th & 25 th & 50 th & 75 th & 90th \\
\hline \multicolumn{6}{|c|}{ Model 2a: Process indicators } \\
\hline experience $_{t}$ & $0.18^{* *}$ & $0.10^{* *}$ & 0.02 & 0.001 & 0.000 \\
\hline experience $_{t} \cdot \operatorname{large}_{i}$ & -0.21 & -0.17 & $-0.19 * *$ & $-0.11^{* * *}$ & $0.000 * * *$ \\
\hline experience $_{t} \cdot$ medium $_{i}$ & $-0.19^{*}$ & $-0.11^{*}$ & -0.03 & -0.01 & 0.000 \\
\hline \multicolumn{6}{|c|}{ Model 2b: Outcome indicators } \\
\hline experience $_{t}$ & $0.86^{* * *}$ & $0.65 * * *$ & $0.41^{* * *}$ & $0.31^{* * *}$ & $0.17 * * *$ \\
\hline experience $_{t} \cdot$ large $_{i}$ & $0.47^{*}$ & 0.12 & -0.19 & $-0.26^{* *}$ & $-0.22^{* * *}$ \\
\hline experience $_{t} \cdot$ medium $_{i}$ & 0.15 & 0.05 & -0.06 & -0.08 & $-0.08^{* *}$ \\
\hline
\end{tabular}

Notes: The columns contain estimated coefficients (in percentage points) from five quantile regressions run for two versions of model 2 . The model $(2 \mathrm{a} / 2 \mathrm{~b})$ regress practice performance in the Quality and Outcomes Framework on experience, experience interacted with the indicator $\operatorname{large}_{i}(\mathrm{BP} 4 / \mathrm{BP} 5)$ and the indicator medium ${ }_{i}(\mathrm{CHD} 5 / \mathrm{CHD} 6)$ (with baseline small $_{i}(\mathrm{STROKE5} / \mathrm{STROKE6})$ ), list size, prevalence rate, prevalence rate interacted with year dummies, exclusion rate, and exception rate. The performance scores are transformed using a logit transformation and adjusted for practice-indicator fixed effects obtained by OLS regression. This table presents the back-transformed estimates (see Appendix $\mathrm{C}$ for the nonback-transformed estimates). Only data for Scottish GPs with a GMS contract for the years 2005 to 2010 are included. Number of observations is 13,986 for both versions of the model. Standard errors are obtained by bootstrapping (400 rep) and reported in Appendix C. ${ }^{*} \mathrm{p}<$ $0.10,{ }^{* *} \mathrm{p}<0.05,{ }^{* * *} \mathrm{p}<0.01$. 
two years of the analysis period. As the two indicators are pivotal for model 2, we instead reduce the analysis period by two years, to assess the robustness of the results from that model. Table 5 (panel III) and table 6 (panel I) show that the results for all models are qualitatively unchanged compared to our main results.

Lastly, we investigate for both model 1 and model 2 whether changes to exception reporting and exclusion reporting by experience may drive our results. We include an interaction term between the exception rate and experience and between the exclusion rate and experience in our two models. As seen from table 5 (panel IV) and table 6 (panel II), for all versions of the two models, the results are qualitatively similar to our previous findings.

\section{Discussion}

Our theoretical analysis shows that as GPs become more certain about their effort-performance relationship they decrease effort above the upper threshold. This result holds except in cases with very high levels of uncertainty. However, the model also predicts that with performance further away from the threshold, the higher is the probability that the GP decreases effort as a response to decreasing uncertainty (see Appendix A). Thus, in the case of the QOF we expect that for a given level of uncertainty, the GPs exerting the largest amount of financially unrewarded performance are expected to respond to a marginally decrease in uncertainty by decreasing effort.

We test our theoretical predictions using data from the GPs' responses to the QOF. As we do not observe a direct measure of GPs' effort and uncertainty, the analysis faces several challenges. In the following we discuss how we have chosen to overcome these challenges and how these choices may affect our results.

\subsection{Measuring effort}

We do not observe physicians' effort level directly. Following other studies on provider behaviour under pay for performance (see for example Kontopantelis et al. (2012); Gravelle et al. (2010)), we use GPs' reported performance on QOF indicators as a proxy for their effort. Research has shown problems with self-reported performance measures at the margin (Gravelle et al., 2010). We acknowledge that misreporting could potentially explain our results, if GPs are more likely to misreport for less certain indicators over time. However, we do not find this likely.

As we define our outcome variable "reported performance" over the interval 0-100\%, our results could be influenced by these limits. When performance on an indicator is close to $100 \%$, the potential for improvement on that indicator is smaller compared to the potential for improvement on an indicator with a lower performance level. Thus, difference in initial performance between indicators could potentially drive differences in performance growth over time and thus drive our results. To address this issue we transform our performance measure using a logit transformation as suggested by Stevens et al. (2016). This transformation linearise performance indicators measured on a percentage scale. In addition, if the bounded nature of the performance range 
Table 5: Robustness of MOdel 1: The EFfeCts OF EXPERIENCE AND INDICATOR TYPE ON PRACTICE REPORTED PERFORMANCE

\begin{tabular}{|c|c|c|c|c|c|}
\hline & \multicolumn{5}{|c|}{ Quantile } \\
\hline & 10 th & 25 th & 50 th & 75 th & 90 th \\
\hline \multicolumn{6}{|c|}{ I: Linearly controlling for the upper threshold level } \\
\hline \multicolumn{6}{|c|}{ Model 1b: All outcome indicators \& process indicators not linked to outcome } \\
\hline experience $_{t}$ & $4.30^{* * *}$ & $2.42^{* * *}$ & $0.54^{* * *}$ & $-0.33^{* * *}$ & $-0.26^{* * *}$ \\
\hline experience $_{t} \cdot$ process $_{i}$ & $-1.85^{* * *}$ & $-1.21^{* * *}$ & $-0.70^{* * *}$ & $-0.32^{* * *}$ & $-0.07^{* * *}$ \\
\hline \multicolumn{6}{|l|}{ Model 1c: All indicators } \\
\hline experience $_{t}$ & $1.62^{* * *}$ & $0.46^{* * *}$ & $-0.11^{* * *}$ & $-0.24^{* * *}$ & $0.00^{* * *}$ \\
\hline experience $_{t} \cdot$ process $_{i}$ & $-1.36^{* * *}$ & $-0.68^{* * *}$ & $-0.35^{* * *}$ & $-0.13^{* * *}$ & $0.00^{* * *}$ \\
\hline \multicolumn{6}{|c|}{ II: Without controlling for the upper threshold level } \\
\hline \multicolumn{6}{|c|}{ Model 1b: All outcome indicators \& process indicators not linked to outcome } \\
\hline experience $_{t}$ & $1.65^{* * *}$ & $1.07^{* * *}$ & $0.49^{* * *}$ & $0.16^{* * *}$ & 0.01 \\
\hline experience $_{t} \cdot$ process $_{i}$ & $-2.55^{* * *}$ & $-1.53^{* * *}$ & $-0.70 * * *$ & $-0.22^{* * *}$ & $-0.01^{*}$ \\
\hline \multicolumn{6}{|l|}{ Model 1c: All indicators } \\
\hline experience $_{t}$ & $1.63^{* * *}$ & $0.70^{* * *}$ & $0.28^{* * *}$ & $0.06^{* * *}$ & 0.00 \\
\hline experience $_{t} \cdot$ process $_{i}$ & $-1.35^{* * *}$ & $-0.59^{* * *}$ & $-0.22^{* * *}$ & $-0.03^{* * *}$ & $0.00^{* * *}$ \\
\hline \multicolumn{6}{|c|}{ III: Excluding indicators CHD06 and BP04 } \\
\hline \multicolumn{6}{|c|}{ Model 1c: All indicators (excl. CHD06 and BP04) } \\
\hline experience $_{t}$ & $1.44^{* * *}$ & $0.59 * * *$ & $0.21^{* * *}$ & $0.04^{* * *}$ & 0.00 \\
\hline experience $_{t} \cdot$ process $_{i}$ & $-1.64^{* * *}$ & $-0.80^{* * *}$ & $-0.39 * * *$ & $-0.14^{* * *}$ & $0.00^{* * *}$ \\
\hline \multicolumn{6}{|c|}{ IV: Including interaction between exception rate/exclusion rate and experience } \\
\hline \multicolumn{6}{|c|}{ Model 1a: Indicators with the same upper threshold level } \\
\hline experience $_{t}$ & $1.22^{* * *}$ & $0.93^{* * *}$ & $0.57^{* * *}$ & $0.36^{* * *}$ & $0.19^{* * *}$ \\
\hline experience $_{t} \cdot$ process $_{i}$ & $-1.91^{* * *}$ & $-1.29 * * *$ & $-0.87 * * *$ & $-0.53^{* * *}$ & $-0.21^{* * *}$ \\
\hline \multicolumn{6}{|c|}{ Model 1b: All outcome indicators \& process indicators not linked to outcome } \\
\hline experience $_{t}$ & $1.72^{* * *}$ & $1.07^{* * *}$ & $0.46^{* * *}$ & $0.18^{* * *}$ & $0.04^{* * *}$ \\
\hline experience $_{t} \cdot$ process $_{i}$ & $-1.87^{* * *}$ & $-1.23^{* * *}$ & $-0.72^{* * *}$ & $-0.35^{* * *}$ & $-0.07^{* * *}$ \\
\hline \multicolumn{6}{|l|}{ Model 1c: All indicators } \\
\hline experience $_{t}$ & $1.60 * * *$ & $0.66^{* * *}$ & $0.24^{* * *}$ & $0.06^{* * *}$ & 0.00 \\
\hline experience $_{t} \cdot$ process $_{i}$ & $-1.63^{* * *}$ & $-0.84^{* * *}$ & $-0.43^{* * *}$ & $-0.16^{* * *}$ & $0.00^{* * *}$ \\
\hline
\end{tabular}

Notes: The columns contain estimated coefficients (in percentage points) from five quantile regressions run for three different versions of model 1 (1a, 1b, and 1c). The models follow the specification used to obtain our main results (see notes to table 3 ) with changes as specified in each header. The numbers of observations are 18,624, 32,574, and 106,902 for model 1a, 1b, and 1c, respectively; except for the model "excluding indicators CHD06 and BP04", which includes 97,596 observations. Standard errors are obtained by bootstrapping (400 rep) ${ }^{*} \mathrm{p}<$ $0.10,{ }^{* *} \mathrm{p}<0.05,{ }^{* * *} \mathrm{p}<0.01$. 
Table 6: Robustness of model 2: The efFects of EXPerience, PATIEnt SCALE And INDICATOR TYPE ON PRACTICE REPORTED PERFORMANCE

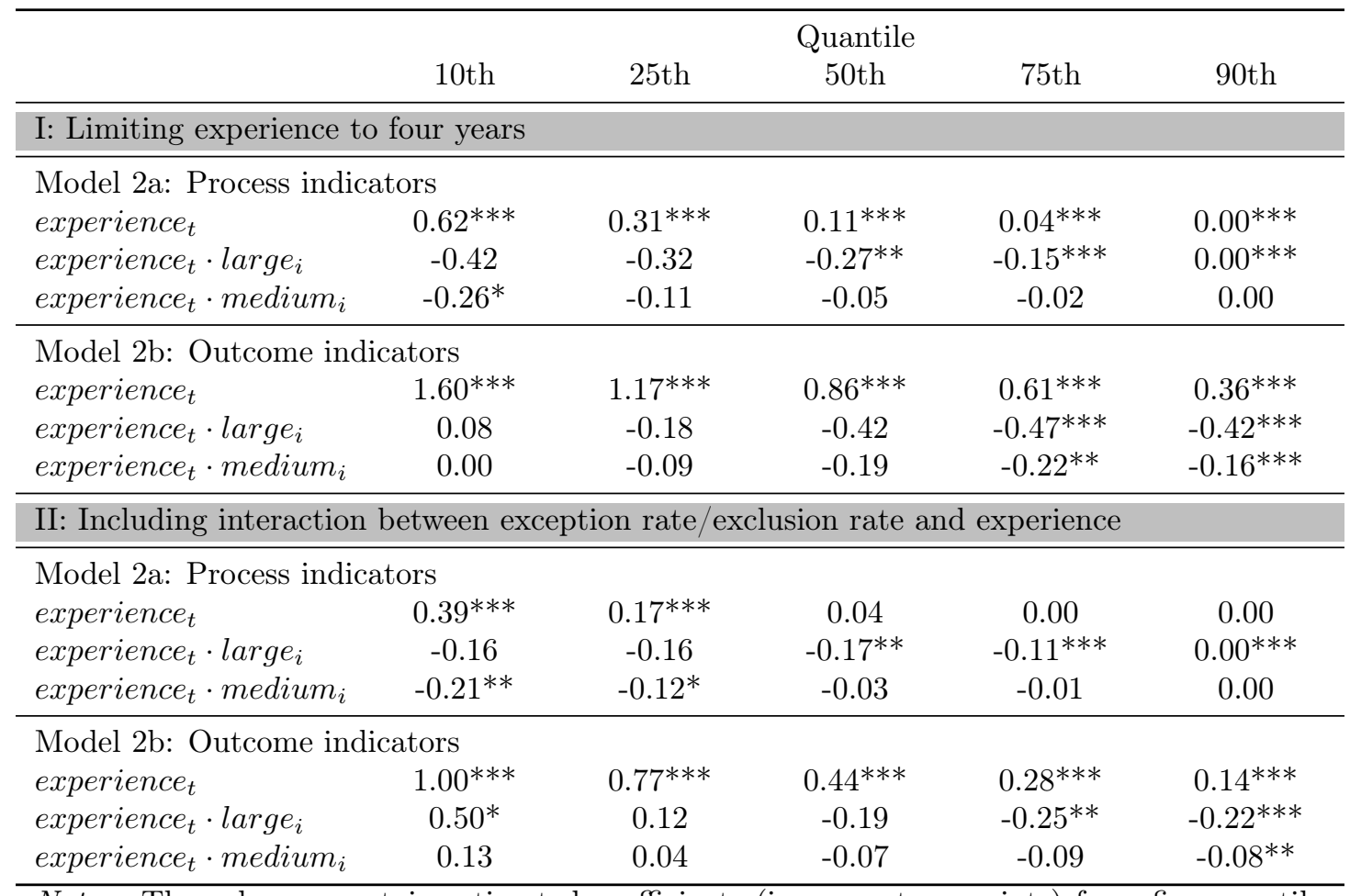

Notes: The columns contain estimated coefficients (in percentage points) from five quantile regressions run for two different versions of model $2(2 \mathrm{a}$ and $2 \mathrm{~b})$. The models follow the specification used to obtain our main results (see notes to table 4) with changes as specified in each header. The number of observations is 9,324 for both model $2 \mathrm{a}$ and $2 \mathrm{~b}$ in the case of limiting analysis to four years of experience. The number of observations is 13,986 for both model $2 \mathrm{a}$ and $2 \mathrm{~b}$ in the case of including interaction between exception rate/exclusion rate and experience. Standard errors are obtained by bootstrapping (400 rep) ${ }^{*} \mathrm{p}<0.10,{ }^{* *} \mathrm{p}<$ $0.05, * * * \mathrm{p}<0.01$. 
were to drive our results, we would expect the initial potential for performance improvement to be greater for the more uncertain indicators, as this might explain the relative decrease in performance over experience for the less uncertain indicators, i.e. our larger-scaled measures and process measures. Yet this is not the case.

In terms of the "scale of eligible patients", we do not observe a larger potential for improvement for the more uncertain indicator relative to the less uncertain indicator. In fact we find the opposite in our model 2: The average performance on the large-scaled (process) indicator in the first year (mean $=93.58 \%$, s.d. $=5.07$ ) is lower than the average performance on the small-scaled (process) indicator (mean $=97.23 \%$, s.d. $=3.56$ ). Thus, the GPs are less able to improve performance over time for the more uncertain indicator, implying that the bounded performance range is not driving our results.

In terms of "indicator type", we observe lower mean performance on outcome indicators compared to process indicators, cf. table 1. This difference in performance is also reflected by a difference in the upper threshold level for the two indicator types. Therefore, we test different versions of model 1. Model 1a only include indicators with a similar upper threshold level of $70 \%$. Comparing GPs' average performance on these indicators the first year of analysis shows that average performance on the process indicator is lower $($ mean $=82.36 \%$, s.d. $=0.41$ ) than average performance on the outcome indicators (mean $=86.09 \%$, s.d. $=0.18$ ), suggesting that our results are not driven by the difference in the potential for improvement.

\subsection{Measuring uncertainty}

As we do not have a direct measure of GPs' uncertainty about the effort-performance relationship, we measure uncertainty by their experience with the scheme and their span of control. In the empirical analysis we include practice-indicator fixed effect to control for differences in GPs' inherent ability or willingness to perform on an indicator. We thereby ensure that differences in neither GPs' inherent ability nor their willingness to perform well on some indicators drive our results.

Our empirical strategy is based on the hypothesis that as GPs gain experience with the scheme, uncertainty decreases more for easily controllable indicators relatively to less easily controllable indicators. It could be argued that the decline in performance over time for process indicators relative to outcome indicators is caused by a decrease in GPs' abilities rather than uncertainty. We have no reason to believe this is the case, as previous studies find that GPs are able to increase performance when an upper threshold for a process indicator in the QOF is increased (Feng et al., 2015; Kontopantelis et al., 2012).

It may also be argued that the decline in performance over time for process indicators relative to outcome indicators may be driven by changes in GPs' altruistic concerns as they gain experience with the scheme. There are reasons to expect that our measures of GPs' span of control may be affected differently by altruistic concerns. If GPs become more altruistic over time, we would expect them to focus more on outcome indicators (uncertain measures) compared to 
process indicators (certain measures), as the outcome indicators relate directly to their patients' health. By contrast, we would expect the GPs to focus more on the larger-scaled indicators (certain measures) than on the smaller-scaled indicators (uncertain measures), as the large-scaled indicators affect a larger patient population. Reassuringly, the two different measures of GPs' span of control yield similar results, indicating that GPs' motives such as altruistic concerns do not explain our results.

As we do not observe data at the patient level, we cannot take into account whether patients may be eligible for multiple indicators. Studies have found that there may be spillover between indicators with similar target groups (see for example Sutton et al. (2010)). Thus, improvement in process indicators may spill over to outcome indicators. In the presence of such spillover effects, practices may exert less control over processes that are strongly correlated with outcomes, as overachievement on the process indicator may be required to achieve the (more uncertain) outcome. We investigate whether this is the case by focusing only on process indicators that are a part of a disease domain with no outcome indicators, cf. model 1b. As expected, we find that the rate of decline in reported performance over experience is significantly higher for indicators not linked to outcomes (model $1 \mathrm{~b}$ versus model 1c).

This paper addresses thresholds based on an absolute performance standard. In such schemes the uncertainty that providers face is caused by the fact that patients do not always comply with treatment and the difficulty for providers in estimating their own achievement. Thus, we assume that providers face uncertainty in the form of a shock to their own performance. Performance thresholds can also be based on relative standards. Relative standards mean that the thresholds are dependent on other providers' performance. This approach introduces a different type of uncertainty in the form of competition. The advantage of applying relative standards is that they automatically take into account common shocks to performance across providers (Lazear and Rosen, 1981). However, introducing competition between providers may also lead to perverse incentives such as a lack of co-operation between providers. As thresholds based on a game between GPs include different incentives than those based on absolute performance, we refrain from generalising our results to these types of schemes.

Overall, we interpret our findings as a confirmation of the hypothesis that uncertainty may plays an important role in physicians' response to an incentive scheme. We focus on the role of uncertainty in pay for performance schemes with an upper threshold based on absolute performance. While our results are specific to this context, this paper is an important step in understanding the role that uncertainty about the effort-performance relationship plays in pay for performance schemes. For future work it would be interesting to investigate the importance of practice characteristics, such as practice size, number of staff employed, and years working as a GP, for their response to uncertainty. 


\section{Conclusion}

In this paper we follow the work by Zhou and Swan (2003) and Oxholm (2016) and propose a theoretical model to explain providers' behaviour when exposed to a performance scheme with no financial payment above a performance threshold. Similar to Oxholm (2016) our model predicts that when providers are uncertain about the effort-performance relationship, those who perform above the upper threshold increase their effort compared to a situation where they can perfectly predict their own performance.

We tested our theoretical predictions using data on the performance of Scottish GPs under the Quality and Outcomes Framework. Our empirical analyses confirm our expectation that as practices gain experience with the scheme, practices performing above the upper threshold reduce achievement on process indicators that are easier to control compared to outcome indicators. For the lower part of the performance distribution the relative decrease in reported performance over the six-year experience period is more than one percentage point per year. Similarly, we find that for indicators with a large scale of eligible patients (less uncertain) the reduction in achievement with experience is larger than for indicators with a small scale of eligible patients. Overall our results support our hypothesis that uncertainty about the effort-performance relationship can explain unrewarded performance.

Our findings suggest that provider uncertainty about the effort-performance relationship is important for explaining financially unrewarded performance. As providers become better at predicting their performance, they decrease their amount of unrewarded performance. Yet providers continue to perform above the upper threshold, suggesting that there may still be an advantage of introducing an upper threshold also over time, if achievement above the threshold is considered a desirable effect. In the case of the QOF we consider an increase in performance above the upper threshold positive, because the scheme offers the option to exclude patients who are ineligible for treatment (Doran et al., 2014).

A way for payers to encourage financially unrewarded performance as providers gain experience with the scheme could be to make the upper threshold dynamic such that providers become more uncertain about the amount of effort required to reach the maximum performance level. This approach may have unwanted consequences for low-performing practices (see Oxholm (2016)), but would be attractive in schemes - like the QOF - where almost all practices initially reaches the upper threshold. In line with this suggestion, the Department of Health in 2013/2014 changed the upper thresholds for some of the clinical indicators in the QOF to the $75 t h$ percentile of the previous year's national performance for each indicator (Doran et al., 2014), making the physicians more uncertain about the required effort in the coming periods. The effect of these changes has yet to be analysed. 


\section{References}

Allen, T., T. Mason, and W. Whittaker (2014, July). Impacts of pay for performance on the quality of primary care. Risk Management and Healthcare Policy 7, 113-120.

Baker, G. (2002). Distortion and risk in optimal incentive contracts. Journal of human resources, $728-751$.

Barnett, A. G., J. C. van der Pols, and A. J. Dobson (2005, February). Regression to the mean: what it is and how to deal with it. International Journal of Epidemiology 34(1), 215-220.

Buetow, S. (2008). Pay-for-performance in new zealand primary health care. Journal of Health Organization and Management 22(1), 36-47.

Cameron, A. C. and P. K. Trivedi (2008). Microeconometrics using stata.

Campbell, S. M., D. Reeves, E. Kontopantelis, B. Sibbald, and M. Roland (2009). Effects of Pay for Performance on the Quality of Primary Care in England. New England Journal of Medicine 361(4), 368-378.

Canay, I. A. (2011). A simple approach to quantile regression for panel data. The Econometrics Journal 14(3), 368-386.

Cashin, C., Y.-L. Chi, P. C. Smith, M. Borowitz, and S. Thomson (2014). Paying for performance in health care: implications for health system performance and accountability. McGraw-Hill Education (UK).

Chalkley, M. and J. M. Malcomson (1998). Contracting for health services when patient demand does not reflect quality. Journal of Health Economics 17(1), 1 - 19.

Chen, J., A. Vargas-Bustamante, K. Mortensen, and S. B. Thomas (2014). Using quantile regression to examine health care expenditures during the great recession. Health services research $49(2), 705-730$.

Cromwell, J., M. G. Trisolini, G. C. Pope, J. B. Mitchell, and L. M. Greenwald (2011). Pay for performance in health care: Methods and approaches, Volume 1. RTI Press.

DepartmentofHealth (2003). Investing in general practice: The new general medical services contract. Technical report.

Dionne, G. and A.-P. Contandriopoulos (1985). Doctors and their workshops: A review article. Journal of Health Economics 4(1), 21 - 33.

Doran, T., C. Fullwood, D. Reeves, H. Gravelle, and M. Roland (2008). Exclusion of patients from pay-for-performance targets by english physicians. New England Journal of Medicine 359(3), $274-284$.

Doran, T., E. Kontopantelis, D. Reeves, M. Sutton, and A. M. Ryan (2014). Setting performance targets in pay for performance programmes: what can we learn from qof. BMJ 348, g1595.

Eggleston, K. (2005). Multitasking and mixed systems for provider payment. Journal of Health Economics 24(1), 211 - 223.

Eijkenaar, F. (2013). Key issues in the design of pay for performance programs. The European Journal of Health Economics 14(1), 117-131. 
Ellis, R. P. and T. G. McGuire (1986). Provider behavior under prospective reimbursement: Cost sharing and supply. Journal of Health Economics 5(2), 129 - 151.

Feng, Y., A. Ma, S. Farrar, and M. Sutton (2015). The tougher the better: an economic analysis of increased payment thresholds on the performance of general practices. Health economics $24(3)$, $353-371$.

Fleetcroft, R. and R. Cookson (2006). Do the incentive payments in the new nhs contract for primary care reflect likely population health gains? Journal of Health Services Research 8 Policy 11 (1), 27-31.

Gillam, S. J., A. N. Siriwardena, and N. Steel (2012). Pay-for-performance in the united kingdom: impact of the quality and outcomes framework - a systematic review. The Annals of Family Medicine 10(5), 461-468.

Giuffrida, A., T. Gosden, F. Forland, I. Kristiansen, M. Sergison, B. Leese, L. Pedersen, and M. Sutton (1999). Target payments in primary care: effects on professional practice and health care outcomes. The cochrane library.

Godager, G. and D. Wiesen (2013). Profit or patients' health benefit? exploring the heterogeneity in physician altruism. Journal of Health Economics 32(6), 1105 - 1116.

Gravelle, H., M. Sutton, and A. Ma (2010). Doctor behaviour under a pay for performance contract: Treating, cheating and case finding? The Economic Journal 120(542), F129-F156.

Guthrie, B., G. McLean, and M. Sutton (2006). Workload and reward in the Quality and Outcomes Framework of the 2004 general practice contract. British Journal of General Practice 56(532), 836-841.

Holmstrom, B. and P. Milgrom (1991). Multitask principal-agent analyses: Incentive contracts, asset ownership, and job design. Journal of Law, Economics, ES Organization 7, 24-52.

ISDScotland (2013, 24 September). Quality and outcomes framework, prevalence, achievement, payment and exceptions data for scotland. Technical report.

Kaarboe, O. and L. Siciliani (2011). Multi-tasking, quality and pay for performance. Health Economics 20(2), 225-238.

Kiran, T., A. S. Wilton, R. Moineddin, L. Paszat, and R. H. Glazier (2014). Effect of payment incentives on cancer screening in ontario primary care. The Annals of Family Medicine 12(4), 317-323.

Kontopantelis, E., T. Doran, H. Gravelle, R. Goudie, L. Siciliani, and M. Sutton (2012). Family doctor responses to changes in incentives for influenza immunization under the u.k. quality and outcomes framework pay-for-performance scheme. Health Services Research $47(3 \mathrm{pt} 1)$, 1117-1136.

Lazear, E. P. and S. Rosen (1981). Rank-order tournaments as optimum labor contracts. Journal of political Economy $89(5), 841-864$.

Makris, M. and L. Siciliani (2013). Optimal incentive schemes for altruistic providers. Journal of Public Economic Theory 15(5), 675-699. 
McLean, G., B. Guthrie, and M. Sutton (2007). Differences in the quality of primary medical care for cvd and diabetes across the nhs: evidence from the quality and outcomes framework. BMC Health Services Research 7(1), 74.

Moscelli, G., L. Siciliani, N. Gutacker, and R. Cookson (2018). Socioeconomic inequality of access to healthcare: Does choice explain the gradient? Journal of health economics 57, 290-314.

Odesjo, H., A. Anell, S. Gudbjornsdottir, J. Thorn, and S. Bjorck (2015). Short-term effects of a pay-for-performance programme for diabetes in a primary care setting: an observational study. Scandinavian journal of primary health care 33(4), 291-297.

Oxholm, A. S. (2016). Physician response to target-based performance payment. COHERE discussion paper (9).

Roland, M. (2004). Linking physicians pay to the quality of care. a major experiment in the united kingdom. N Engl J Med 2004(351), 1448-1454.

Scott, A., M. Liu, and J. Yong (2018). Financial incentives to encourage value-based health care. Medical Care Research and Review 75(1), 3-32.

Stevens, S., J. M. Valderas, T. Doran, R. Perera, and E. Kontopantelis (2016). Analysing indicators of performance, satisfaction, or safety using empirical logit transformation. bmj 352, i1114.

Sutton, M., R. Elder, B. Guthrie, and G. Watt (2010). Record rewards: the effects of targeted quality incentives on the recording of risk factors by primary care providers. Health Economics 19(1), 1-13.

Zhou, X. and P. L. Swan (2003). Performance thresholds in managerial incentive contracts. The Journal of Business 76(4), 665-696. 


\section{A Changes in uncertainty's effect on GP's optimal effort level}

Following Oxholm (2016) we assume that GP $g$ is uncertain about the effort-performance relationship. Let uncertainty be characterised by a random shock to performance, which is normally distributed with mean zero, i.e. $N \sim\left(0, \sigma^{2}\right)$. Recall that the GP has no expected utility gain from increasing effort when having found the optimal effort level:

$$
h_{g}^{\prime}\left(e_{g}\right)+\beta \int_{-\infty}^{\tau-e_{g}} \phi(\varepsilon) d \varepsilon=c_{g}^{\prime}\left(e_{g}\right)
$$

If uncertainty rises, the GP's marginal utility gain from effort associated with the performance payment is the only variable that is altered. The GP's marginal utility gain from effort associated with the performance payment is thus given by the probability distribution function of the normal distribution:

$$
\phi(\varepsilon)=\frac{1}{\sigma \sqrt{2 \pi}} e^{\frac{-\varepsilon^{2}}{2 \sigma^{2}}}
$$

Now let the variance of the shock increase marginally. This increase affects the GP's marginal utility gain from effort associated with the performance payment:

$$
\frac{\partial\left(\beta \int_{-\infty}^{\tau-e_{g}} \phi(\varepsilon) d \varepsilon\right)}{\partial \sigma}=\beta \int_{-\infty}^{\tau-e_{g}}\left(\frac{\varepsilon^{2}}{\sigma^{2}}-1\right) \frac{\sqrt{2} e^{\frac{-\varepsilon^{2}}{2 \sigma^{2}}}}{2 \sigma^{2} \sqrt{\pi}} d \varepsilon \lesseqgtr 0
$$

The change in uncertainty can both affect expected gain from exerting effort positively and negatively. It depends on three factors: The GP's initial degree of uncertainty, $\sigma^{2}$, the GP's initial effort level, $e_{g}$, and the threshold effort level, $\tau$. The results are similar to the ones found in Oxholm (2016) and imply that the further away from the threshold level the GP initially exerts effort, the more likely it is that a decrease in the variance of a shock decreases the marginal utility of exerting effort. Furthermore, the lower the initial degree of uncertainty is the higher is the probability that a decrease in the variance decreases the marginal utility of exerting effort. 


\section{B Estimation results for model 1 with non-back transformed performance scores}

Table B1: THE EFFECTS OF EXPERIENCE AND INDICATOR TYPE ON PRACTICE REPORTED TRANSFORMED PERFORMANCE

\begin{tabular}{|c|c|c|c|c|c|}
\hline & \multicolumn{5}{|c|}{ Quantile } \\
\hline & 10th & 25th & 50 th & 75th & 90 th \\
\hline \multicolumn{6}{|c|}{ Model 1a: Indicators with the same upper threshold level } \\
\hline \multirow[t]{2}{*}{ experience $_{t}$} & $0.064^{* * *}$ & $0.058^{* * *}$ & $0.051^{* * *}$ & $0.050^{* * *}$ & $0.040^{* * *}$ \\
\hline & $(0.006)$ & $(0.006)$ & $(0.005)$ & $(0.006)$ & $(0.007)$ \\
\hline \multirow[t]{2}{*}{ experience $_{t} \cdot$ process $_{i}$} & $-0.107 * * *$ & $-0.091^{* * *}$ & $-0.086^{* * *}$ & $-0.084^{* * *}$ & $-0.067^{* * *}$ \\
\hline & $(0.008)$ & $(0.008)$ & $(0.007)$ & $(0.008)$ & $(0.011)$ \\
\hline Pseudo $R^{2}$ & 0.1713 & 0.1904 & 0.2051 & 0.2053 & 0.2116 \\
\hline Quantile performance & $75.74 \%$ & $81.67 \%$ & $87.90 \%$ & $92.91 \%$ & $96.30 \%$ \\
\hline
\end{tabular}

Notes: The columns contain estimated coefficients from five quantile regressions run for model 1a. The model regresses practice reported performance in the Quality and Outcomes Framework on experience, experience interacted with a dummy equalling one for process indicator, list size, prevalence, prevalence rate interacted with year dummies, exclusion rate, and exception rate. The performance scores are transformed using a logit transformation and adjusted for practice-indicator fixed effects obtained by OLS regression. The model includes the indicators listed in table 1 with an upper threshold level of 70\%. Only data for Scottish GPs with a GMS contract for the years 2005 to 2010 are included. The number of observations are 18,624. Standard errors in parentheses are obtained by bootstrapping (400 rep). ${ }^{*} \mathrm{p}<$ $0.10,{ }^{* *} \mathrm{p}<0.05,{ }^{* * *} \mathrm{p}<0.01$. 
Table B2: The EFFECTS OF EXPERIEnCE AND INDICATOR TYPE ON PRACTICE REPORTED TRANSFORMED PERFORMANCE

\begin{tabular}{lccccc}
\hline & \multicolumn{4}{c}{ Quantile } \\
& 10 th & 25 th & 50 th & 75 th & 90 th \\
\hline Model 1b: All outcome indicators \& process indicators not linked to outcome \\
\hline experience $_{t}$ & $0.081^{* * *}$ & $0.064^{* * *}$ & $0.051^{* * *}$ & $0.043^{* * *}$ & $0.025^{* * *}$ \\
& $(0.006)$ & $(0.005)$ & $(0.005)$ & $(0.006)$ & $(0.007)$ \\
experience $_{t} \cdot$ process $_{i}$ & $-0.109^{* * *}$ & $-0.091^{* * *}$ & $-0.085^{* * *}$ & $-0.082^{* * *}$ & $-0.061^{* * *}$ \\
& $(0.008)$ & $(0.008)$ & $(0.008)$ & $(0.008)$ & $(0.010)$ \\
experience $_{t} \cdot$ upper $_{60 i}$ & $0.024^{* * *}$ & $0.019^{* * *}$ & 0.007 & $-0.019^{* * *}$ & $-0.045^{* * *}$ \\
& $(0.007)$ & $(0.007)$ & $(0.007)$ & $(0.007)$ & $(0.008)$ \\
experience $_{t} \cdot$ upper $_{85 i}$ & $0.037^{* * *}$ & $0.040^{* * *}$ & $0.060^{* * *}$ & $0.070^{* * *}$ & $0.073^{* * *}$ \\
& $(0.011)$ & $(0.011)$ & $(0.011)$ & $(0.011)$ & $(0.013)$ \\
experience $_{t} \cdot$ upper $_{90 i}$ & $-0.083^{* * *}$ & $-0.059^{* * *}$ & $-0.022^{* *}$ & 0.013 & $0.030^{* *}$ \\
& $(0.012)$ & $(0.012)$ & $(0.011)$ & $(0.012)$ & $(0.014)$ \\
\hline Pseudo $^{2}$ & 0.2475 & 0.2292 & 0.2215 & 0.2188 & 0.2144 \\
Quantile performance $^{2 *}$ & $76.39 \%$ & $82.84 \%$ & $90.24 \%$ & $95.51 \%$ & $98.77 \%$ \\
\hline
\end{tabular}

Notes: The columns contain estimated coefficients from five quantile regressions run for model 1b. The model regresses practice reported performance in the Quality and Outcomes Framework on experience, experience interacted with a dummy equalling one for process indicator, list size, prevalence, prevalence rate interacted with year dummies, exclusion rate, and exception rate. In addition, the model controls for experience interacted with dummies for the size of the upper threshold level (baseline 70\%). The performance scores are transformed using a logit transformation and adjusted for practice-indicator fixed effects obtained by OLS regression. The model includes all outcome indicators and process indicators not linked to outcome indicators within a disease domain (ASTHMA06, COPD08, THYROI02) listed in table 1. Only data for Scottish GPs with a GMS contract for the years 2005 to 2010 are included. The number of observations are 32,574. Standard errors in parentheses are obtained by bootstrapping (400 rep). ${ }^{*} \mathrm{p}<0.10,{ }^{* *} \mathrm{p}<0.05,{ }^{* * *} \mathrm{p}<0.01$. 
Table B3: THE EFFECTS OF EXPERIENCE AND INDICATOR TYPE ON PRACTICE REPORTED TRANSFORMED PERFORMANCE

\begin{tabular}{lccccc}
\hline & & & Quantile & \\
& 10 th & 25 th & 50 th & 75 th & 90 th \\
\hline Model 1c: All indicators & & & & \\
experience & & & & & \\
& $0.111^{* * *}$ & $0.082^{* * *}$ & $0.059^{* * *}$ & $0.039^{* * *}$ & 0.009 \\
experience $_{t} \cdot$ process $_{i}$ & $(0.005)$ & $(0.005)$ & $(0.005)$ & $(0.005)$ & $(0.006)$ \\
& $-0.111^{* * *}$ & $-0.095^{* * *}$ & $-0.087^{* * *}$ & $-0.083^{* * *}$ & $-0.060^{* * *}$ \\
experience $_{t} \cdot$ upper $_{60 i}$ & $(0.009)$ & $(0.008)$ & $(0.008)$ & $(0.008)$ & $(0.010)$ \\
& $0.012^{*}$ & 0.008 & 0.000 & $-0.024^{* * *}$ & $-0.043^{* * *}$ \\
experience $_{t} \cdot$ upper $_{85 i}$ & $(0.006)$ & $(0.006)$ & $(0.006)$ & $(0.007)$ & $(0.007)$ \\
& $0.045^{* * *}$ & $0.048^{* * *}$ & $0.061^{* * *}$ & $0.072^{* * *}$ & $0.076^{* * *}$ \\
experience $_{t} \cdot$ upper $_{90 i}$ & $(0.010)$ & $(0.009)$ & $(0.009)$ & $(0.009)$ & $(0.011)$ \\
& $0.018^{* *}$ & $0.026^{* * *}$ & $0.042^{* * *}$ & $0.060^{* * *}$ & $0.071^{* * *}$ \\
& $(0.009)$ & $(0.008)$ & $(0.007)$ & $(0.008)$ & $(0.009)$ \\
\hline Pseudo $^{2}$ & 0.1800 & 0.1699 & 0.1691 & 0.1629 & 0.1578 \\
Quantile performance $^{2 *}$ & $83.09 \%$ & $90.91 \%$ & $95.14 \%$ & $98.19 \%$ & $100 \%$ \\
\hline
\end{tabular}

Notes: The columns contain estimated coefficients from five quantile regressions run for model 1c. The model regresses practice reported performance in the Quality and Outcomes Framework on experience, experience interacted with a dummy equalling one for process indicator, list size, prevalence, prevalence rate interacted with year dummies, exclusion rate, and exception rate. In addition, the model controls for experience interacted with dummies for the size of the upper threshold level (baseline 70\%). The performance scores are transformed using a logit transformation and adjusted for practice-indicator fixed effects obtained by OLS regression. The model includes the indicators listed in table 1. Only data for Scottish GPs with a GMS contract for the years 2005 to 2010 are included. The number of observations are 106,902. Standard errors in parentheses are obtained by bootstrapping (400 rep). ${ }^{*} \mathrm{p}<$ $0.10,{ }^{* *} \mathrm{p}<0.05,{ }^{* * *} \mathrm{p}<0.01$. 


\section{Estimation results for model 2 with non-back transformed performance scores}

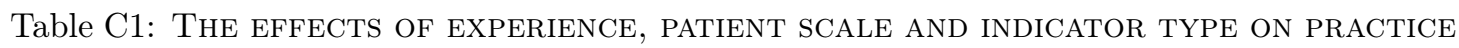
REPORTED PERFORMANCE

\begin{tabular}{lccccc}
\hline & & & Quantile & & \\
& 10 th & 25 th & 50 th & 75 th & 90 th \\
\hline Model 2a: Process indicators & $0.026^{* *}$ & $0.021^{* *}$ & 0.009 & 0.001 & -0.017 \\
experience $_{t}$ & $(0.013)$ & $(0.010)$ & $(0.010)$ & $(0.011)$ & $(0.012)$ \\
experience $_{t} \cdot$ large $_{i}$ & -0.028 & -0.033 & $-0.071^{* *}$ & $-0.120^{* * *}$ & $-0.169^{* * *}$ \\
& $(0.035)$ & $(0.030)$ & $(0.028)$ & $(0.026)$ & $(0.034)$ \\
experience $_{t} \cdot$ medium $_{i}$ & $-0.026^{*}$ & $-0.022^{*}$ & -0.011 & -0.013 & -0.010 \\
& $(0.014)$ & $(0.012)$ & $(0.012)$ & $(0.013)$ & $(0.014)$ \\
\hline Pseudo $^{2}$ & 0.0338 & 0.0467 & 0.0797 & 0.0912 & 0.0888 \\
Quantile performance $^{2}$ & $92.16 \%$ & $94.73 \%$ & $97.40 \%$ & $99.13 \%$ & $100 \%$ \\
\hline Notes: The
\end{tabular}

Notes: The columns contain estimated coefficients from five quantile regressions run for model 2a. The model regress practice performance in the Quality and Outcomes Framework on experience, experience interacted with the indicator large $_{i}(\mathrm{BP} 4)$, medium $_{i}(\mathrm{CHD} 5)$ (and baseline small $_{i}\left(\mathrm{STROKE}_{5}\right)$ ), list size, prevalence rate, prevalence rate interacted with year dummies, exclusion rate, and exception rate. The performance scores are transformed using a logit transformation and adjusted for practice-indicator fixed effects obtained by OLS regression. Only data for Scottish GPs with a GMS contract for the years 2005 to 2010 are included. Number of observations is 13,986 . Standard errors in parentheses are obtained by bootstrapping (400 rep). ${ }^{*} \mathrm{p}<0.10,{ }^{* *} \mathrm{p}<0.05,{ }^{* * *} \mathrm{p}<0.01$. 


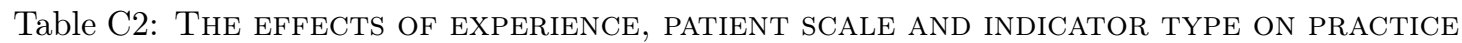
REPORTED PERFORMANCE

\begin{tabular}{lccccc}
\hline & & & Quantile & & \\
& 10 th & 25 th & 50 th & 75 th & 90 th \\
\hline Model 2b: Outcome indicators $^{*}$ & $0.051^{* * *}$ & $0.0485^{* * *}$ & $0.043^{* * *}$ & $0.051^{* * *}$ & $0.052^{* * *}$ \\
& $(0.007)$ & $(0.007)$ & $(0.007)$ & $(0.007)$ & $(0.009)$ \\
experience $_{t}$ & $0.0280^{*}$ & 0.009 & -0.020 & $-0.040^{* *}$ & $-0.063^{* * *}$ \\
experience $_{t} \cdot$ large $_{i}$ & $(0.170)$ & $(0.017)$ & $(0.017)$ & $(0.017)$ & $(0.018)$ \\
experience $_{t} \cdot$ medium $_{i}$ & 0.009 & 0.004 & -0.007 & -0.013 & $-0.022^{* *}$ \\
& $(0.009)$ & $(0.009)$ & $(0.008)$ & $(0.010)$ & $(0.011)$ \\
\hline${\text { Pseudo } R^{2}}^{\text {Quantile performance }}$ & 0.1080 & 0.0884 & 0.0767 & 0.0923 & 0.1299 \\
\hline
\end{tabular}

Notes: The columns contain estimated coefficients from five quantile regressions run for model $2 \mathrm{~b}$. The model regress practice performance in the Quality and Outcomes Framework on experience, experience interacted with the indicator large $_{i}(\mathrm{BP} 5)$, medium $_{i}$ (CHD6) (and baseline small $_{i}($ STROKE6)), list size, prevalence rate, prevalence rate interacted with year dummies, exclusion rate, and exception rate. The performance scores are transformed using a logit transformation and adjusted for practice-indicator fixed effects obtained by OLS regression. Only data for Scottish GPs with a GMS contract for the years 2005 to 2010 are included. Number of observations is 13,986 . Standard errors in parentheses are obtained by bootstrapping (400 rep). ${ }^{*} \mathrm{p}<0.10,{ }^{* *} \mathrm{p}<0.05,{ }^{* * *} \mathrm{p}<0.01$. 